Pacific Journal of Mathematics

BOUNDS FOR EIGENVALUES OF THE FREE AND FIXED 


\title{
BOUNDS FOR EIGENVALUES OF THE FREE AND FIXED MEMBRANE BY FINITE DIFFERENCE METHODS
}

\author{
BERT HUBBARD
}

\section{CHAPTER I}

Introduction. This paper gives explicit upper and lower bounds for the eigenvalues of both the free and fixed membrane problems in terms of the eigenvalues of analogous finite difference problems. For the fixed membrane we seek eigenvalues of the Laplace operator on a bounded region $R$ of the Euclidean plane under the added condition that the solution must vanish on the boundary $C$. In the case of the free membrane it is the normal derivative which vanishes on $C$. If $C$ is sufficiently smooth the difference between the upper and lower bounds is of the order of the grid width $h$.

Generally upper and lower bounds for the membrane eigenvalues, $\lambda_{1} \leqq \lambda_{2} \leqq \cdots$, have been obtained separately and by distinctly different methods. Upper bounds for $\lambda_{k}$ can always be obtained by the RayleighRitz process [53]. This depends on the minimum-maximum property of $\lambda_{k}$ which was first discovered by Poincaré [46]. Following a suggestion of R. Courant [13] (also appearing implicitly in a paper of L. Collatz [8]) we can express this upper bound in terms of the eigenvalues of a related finite difference problem. Such upper bounds have been obtained by G. Pólya [47] and H. Weinberger [67] for particular finite difference analogues of the fixed membrane.

Lower bounds, in general, present a more formidable problem. In this case the maximum-minimum property [12] of $\lambda_{k}$ is usually exploited. Perhaps the best known method is that of A. Weinstein [71] which gives arbitrarily close lower bounds for a symmetric linear elliptic differential operator $A$ defined on a subspace $V$ of a Hilbert space $H$. This method presupposes a knowledge of the eigenvalues and eigenvectors of an extension $A^{\prime}$ of $A$ to a space $V^{\prime}$ which contains $V$. The method of A. Weinstein has been extended by $N$. Aronszajn [1, 2] and N. Bazley [3, $4,5]$.

Certain methods for obtaining lower bounds have been pointed out by E. Trefftz [58], G. Temple [57], T. Kato [28], and others. Many of these and other methods are summarized in an excellent survey by $\mathrm{J}$. B. Diaz [15]. Many of these have been unified into a single theory in

Received May 24, 1960. Submitted in partial fulfillment of the requirements for the degree of Doctor of Philosophy in the Department of Mathematics, University of Maryland. This work was carried out at the Naval Ordnance Laboratory under the sponsorship of the Foundational Research Program. 
a recent paper of $\mathrm{H}$. Weinberger [69]. In order to apply any of the methods mentioned it is necessary to have already an exact lower bound for at least one of the higher eigenvalues of $A$. The finite difference methods presented here require no such additional knowledge.

The Poincaré inequality can also be used to obtain upper bounds for the eigenvalues, $\mu_{1}(h) \leqq \mu_{2}(h) \leqq \cdots$, of a suitably defined finite difference problem. If the vectors in this inequality are appropriately defined in terms of the first $k$ eigenfunctions of the membrane problem it is possible to relate this upper bound for $\mu_{k}(h)$ to $\lambda_{k}$. The resulting inequality is then solved to give a lower bound for $\lambda_{k}$ in terms of $\mu_{k}(h)$. This technique has been applied by L. Collatz [8], G. Forsythe $[19,20]$, J. Hersch [24], and H. Weinberger [67]. The present paper relies heavily on the results of the latter author.

In Chapter II a finite difference eigenvalue problem is posed for the fixed membrane. Both upper and lower bounds for $\lambda_{k}$ are obtained terms of $\mu_{k}(h)$ and quantities involving the geometry of the boundary $C$. Most of the effort is expended in bounding a certain integral defined over a thin strip of $R$ in the neighborhood of the boundary. Once this is accomplished the difference between the upper and lower bound is explicitly bounded by a term of the order of the mesh size, $h$. In the papers alluded to above, distinct finite difference problems were formulated to give upper and lower bounds. In most cases the difference between the upper and lower bounds was not explicitly bounded. It is known however, that the eigenvalues of such finite difference problems converge to the membrane eigenvalues as $h \rightarrow 0$. Clearly, by posing only one matrix eigenvalue problem we cut the actual computation involved in obtaining bounds in half.

In Chapter III bounds for the eigenvalues of the free membrane problem are obtained in a similar manner. Again the difference between the upper and lower bounds is bounded by a term of order $h$. Lower bounds for the free membrane eigenvalues by finite difference methods appear to have been left relatively unexplored. In this problem the eigenvalues do not have a monotone dependence on the region as is the case with fixed membrane eigenvalues. Hence it is difficult to obtain a lower bound for some higher eigenvalue; a requirement for most methods of finding lower bounds. Therefore, this result appears to mark a much greater advance than that obtained in the case of the fixed membrane.

The error bounds depend upon the choice of a vector field defined on $R+C$ subject to weak conditions with one possible method of choosing such a field illustrated in Appendix A. In Appendix B certain parameters depending on the geometry of $C$ are bounded. Finally, the bounds of this paper are computed in two cases for which the solution is known explicitly; (a) the unit square, and (b) the unit circle. They are then 
compared with the actual quantities being bounded.

I wish to express my gratitude to Professor H. F. Weinberger for his valuable assistance in directing my researches into this subject.

CHAPTER II

\section{THE FIXED MEMBRANE PROBLEM}

1. The problem and its finite difference analogue. Let $R$ be a bounded, simply connected region in the $x-y$ plane with boundary $C$. The boundary itself is assumed to be composed of piecewise smooth arcs with interior angles at corners of $C$ which are less than $180^{\circ}$.

Denote the eigenvalues of the fixed membrane problem

$$
\begin{aligned}
\Delta u+\lambda u=0 & \text { in } R, \\
u=0 & \text { on } C,
\end{aligned}
$$

by $\lambda_{1} \leqq \lambda_{2} \leqq \cdots$. The eigenfunctions $u_{1}, u_{2}, \cdots$ are normalized by

$$
\iint_{R} u_{i}^{2} d x d y=1
$$

Divide the $x-y$ plane into squares of width $h$ by the two families of lines $x=m h$ and $y=n h ; m, n=1,2,3, \cdots$. The dependent variables of the finite difference problem are defined at certain of the intersections of these lines, called "mesh points". Superposing a third family of lines of slope 1 through the mesh points divides the plane into isosceles right triangles so that each mesh point has six "associated" triangles of which

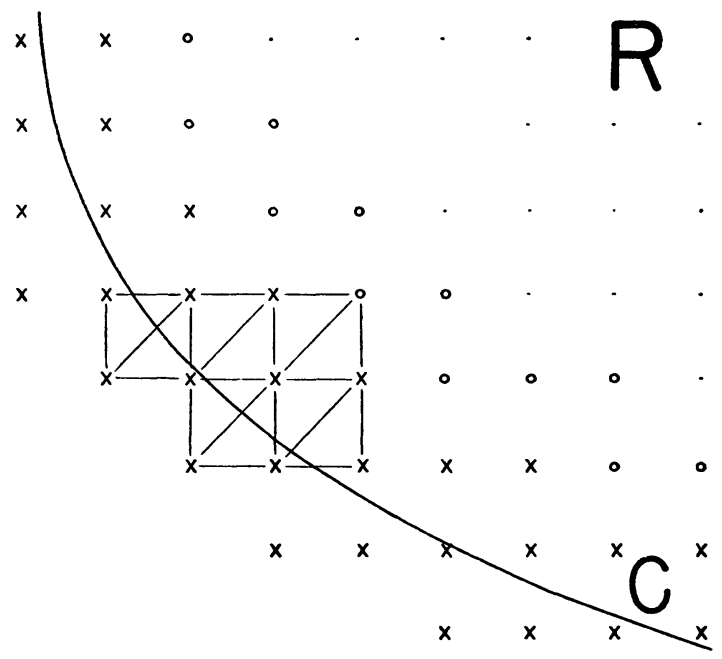

Fig. 1. Symbol Set to which point belongs

$\begin{array}{cc}\times & C_{h} \\ \circ & B_{h} \\ \cdot & R_{h}-c_{h}\end{array}$


it is a vertex. $C_{h}$, is defined to be the set of those mesh points which have at least one associated triangle intersecting $C . \quad R_{h}$ is the collection of mesh points of $C_{h}$. The set $R_{h}-C_{h}$ is composed of those points of $R_{h}$ which do not belong to $C_{h}$, i.e., interior points whose triangles do not cut $C$, (see Fig. 1). In what follows the "mesh functions" are assumed to be defined only at points of $R_{h}$.

The eigenvalues of 2.1 are to be approximated by the eigenvalues $\mu_{1}(h) \leqq \mu_{2}(h) \leqq \cdots$ of the finite difference problem

$$
\begin{aligned}
\Delta_{h} V+\mu(h) V=0 & \text { on } R_{h}-C_{h}, \\
V & =0 \quad \text { on } C_{h},
\end{aligned}
$$

where $V(m, n)$ is a mesh function evaluated at the point $(m h, n h)$ of the plane. Here, $\Delta_{h}$ is the centered second order difference operator

$$
\begin{aligned}
\Delta_{h} V(m, n)= & h^{-2}[V(m+1, n)+V(m, n+1)+V(m-1, n) \\
& +V(m, n-1)-4 V(m, n)] .
\end{aligned}
$$

Let $V_{1}, V_{2}, \cdots$ be the eigenvectors of 2.3 with the normalization

$$
h^{2} \sum_{R_{h}-C_{h}} V_{i}^{2}=1 \text {. }
$$

The eigenvalue $\mu_{k}(h)$ can be obtained as a solution of the minimummaximum problem

$$
\mu_{k}(h)=\min _{W_{1} \cdots W_{k}} \max _{a_{1} \cdots a_{k}} \frac{D_{R_{h}}^{(h)}\left(a_{1} W_{1}+\cdots+a_{k} W_{k}\right)}{h^{2} \sum_{R_{h}-o_{h}}\left(a_{1} W_{1}+\cdots+a_{k} W_{k}\right)^{2}} .
$$

The vectors $W_{1}, \cdots, W_{k}$ are linearly independent mesh functions which vanish at points of $C_{h}$ and $a_{1}, \cdots, a_{k}$ are real numbers. The numerator of the above Rayleigh quotient is given as

$$
\begin{aligned}
D_{R_{h}}^{(h)}(W)=\sum_{R_{h}}\{[W(m & +1, n)-W(m, n)]^{2} \\
& \left.+[W(m, n+1)-W(m, n)]^{2}\right\},
\end{aligned}
$$

where the sum is over all differences of neighboring points of $R_{h}$.

The eigenvalues of the fixed membrane are defined by

$$
\lambda_{k}=\min _{v_{1} \cdots v_{k}} \max _{a_{1} \cdots a_{k}} \frac{D\left(a_{1} v_{1}+\cdots+a_{k} v_{k}\right)}{\iint_{R}\left(a_{1} v_{1}+\cdots+a_{k} v_{k}\right)^{2} d x d y},
$$

where $v_{1}, \cdots, v_{k}$ are also linearly independent, piecewise continuously differentiable functions in $R$ which vanish on $C$ and $D(v)$ is the Dirichlet integral 


$$
D(v)=\iint_{R}\left(v_{x}^{2}+v_{y}^{2}\right) d x d y .
$$

The procedure for obtaining upper bounds for $\lambda_{k}$ in terms of $\mu_{k}$ is to define $k$ admissible functions for the inequality obtained from 2.8 in terms of the first $k$ eigenvectors of the discrete problem. Inequalities are further developed for the numerator and denominator of 2.8 in terms of like quantities of 2.6. The indicated maximization with respect to the real numbers $a_{1}, \cdots, a_{k}$ is then effected to achieve the bound. An upper bound for $\mu_{k}(h)$ is found in a similar manner. This in turn can be solved by using the upper bound for $\lambda_{k}$ to yield a lower bound for $\lambda_{k}$.

2. The upper bound. Starting with the mesh eigenfunctions $V_{1}$, $\cdots, V_{k}$ of the finite difference problem, we define functions $v_{1}, \cdots, v_{k}$ admissible in the continuous problem 2.8 as suggested by L. Collatz [8] and R. Courant [13], (see also G. Pólya [47]). Let $v_{i}(x, y)$ be the piecewise continuously differentiable function which is linear in each triangle and coincides with the eigenvector $V_{i}$ at the vertices. Clearly the linear independence of $v_{1}, \cdots, v_{k}$ assures the linear independence of $v_{1}, \cdots$, $v_{k}$ and the vanishing of each $V_{i}$ at points of $C_{k}$ causes each $v_{i}$ to be zero on $C$. Therefore these functions are admissible in the inequality

$$
\lambda_{k} \leqq \max _{a_{1} \cdots a_{k}} \frac{D(v)}{\iint_{R} v^{2} d x d y},
$$

obtained from 2.8 where

$$
\begin{gathered}
v=a_{1} v_{1}+\cdots+a_{k} v_{k}, \\
V=a_{1} V_{1}+\cdots+a_{k} V_{k} .
\end{gathered}
$$

We next obtain inequalities for the numerator and denominator of 2.10 in terms of $D_{R_{h}}^{(h)}(V)$ and $h^{2} \sum V^{2}$. It is easily shown (see [67], page 361) that

$$
D(v) \leqq D_{R_{h}}^{(h)}(V)
$$

and further that

$$
\begin{aligned}
& \iint_{R} v^{2} d x d y \geqq h^{2} \sum_{R_{h}-\sigma_{h}} V(m, n)^{2}-\frac{h^{2}}{12} \sum_{R_{h}}\left\{[V(m+1, n)-V(m, n)]^{2}\right. \\
& \quad+[V(m, n+1)-V(m, n)]^{2}+\left[(V(m+1, n+1)-V(m, n)]^{2}\right\}, \\
& \quad \geqq h^{2} \sum_{R_{h}-\sigma_{h}} V^{2}-\frac{h^{2}}{4} D_{R_{h}}^{(h)}(V) .
\end{aligned}
$$

Applying these inequalities to 2.10 yields the known upper bound (c.f. Weinberger [67]) 


$$
\begin{gathered}
\lambda_{k} \leqq \max _{a_{1} \cdots a_{k}} \frac{D_{R_{h}}^{(h)}(V)}{h^{2} \sum_{R_{h}-C_{h}} V^{2}-\frac{h^{2}}{4} D_{R_{h}}^{(h)}(V)}, \\
=\max _{a_{1} \cdots a_{k}} \frac{\sum_{i=1}^{k} a_{i}^{2} \mu_{i}(h)}{1-\frac{h^{2}}{4} \sum_{i=1}^{k} a_{i}^{2} \mu_{i}(h)} \leqq \frac{\mu_{k}(h)}{1-\frac{h^{2}}{4} \mu_{k}(h)} .
\end{gathered}
$$

We have chosen a finite difference problem which yields a particularly simple expression for the upper bound. This increases the labor involved in finding the lower bound as we shall see.

3. The lower bound. The following technique was used by Weinberger [67] to find lower bounds for $\lambda_{k}$ in terms of the eigenvalues of a slightly different finite difference problem. The bound obtained in [67] is that of 2.27 with the integral 2.28 omitted.

Extend $u_{i}$ as 0 outside $R$ and let the mesh functions $W_{1}, \cdots, W_{k}$ be defined in terms of the eigenfunctions $u_{1}, \cdots, u_{k}$ as follows:

$$
W_{i}(m, n)=\left\{\begin{array}{cl}
h^{-2} \iint_{S(m, n)} u_{i} d x d y, & (m, n) \in R_{h}-C_{h} \\
0 & (m, n) \in C_{h},
\end{array}\right.
$$

where $S(m, n)$ is the square with center $(m, n)$ and sides of length $h$ oriented in the directions of the $x-y$ axes. Let

$$
\begin{aligned}
W & =a_{1} W_{1}+\cdots+a_{k} W_{k}, \\
u & =a_{1} u_{1}+\cdots+a_{k} u_{k} .
\end{aligned}
$$

Deferring for the moment the question of linear independence we see that in other respects the functions $W_{1}, \cdots, W_{k}$ are admissible in the inequality

$$
\mu_{k}(h) \leqq \max _{a_{1} \cdots a_{k}} \frac{D_{R_{h}}^{(h)}(W)}{h^{2} \sum_{R_{h}-c_{h}} W^{2}}
$$

which arises from 2.6.

We now seek inequalities relating the numerator and denominator of 2.17 to $D(u)$ and $\iint u^{2} d x d y$. It is easily seen that if both $(m, n)$ and $(m+1, n) \in R_{h}$ then

(2.18) $W(m+1, n)-W(m, n)=h^{-2} \int_{0}^{2 h} d \xi \int_{0}^{h} d \gamma \psi(\xi) \frac{\partial u}{\partial x}(m h+\xi, n h+\gamma)$,

with a similar formula for $y$-differences. Here we have put 


$$
\psi(\xi)=\left\{\begin{array}{ll}
\xi & , 0 \leqq \xi \leqq h \\
2 h-\xi, & h \leqq \xi \leqq 2 h
\end{array} .\right.
$$

In the mixed case, e.g. when $(m-1, n) \in C_{h},(m, n) \in R_{h}-C_{h}$, an application of Schwarz's inequality yields

$$
[W(m, n)-W(m-1, n)]^{2}=W(m, n)^{2} \leqq h^{-2} \iint_{S(m, n)} u^{2} d x d y .
$$

We see that $(m, n)$ might have points of $C_{h}$ on as many as three sides with the result that $W(m, n)^{2}$ could appear with a factor of three in the Dirichlet sum, $D_{R_{h}}^{(h)}(W)$. If $B_{h}$ is defined to be the set of those points of $R_{h}-C_{h}$ adjacent to points of $C_{h}$ (see Fig. 1), then

$$
D_{R_{h}}^{(h)}(W) \leqq 3 \sum_{B_{h}} W^{2}+D_{R_{h}-C_{h}}^{(h)}(W) .
$$

A computation using the representation 2.18 shows that

$$
\begin{aligned}
& \left.D(u)-D_{R_{h}-\sigma_{h}}^{(h)}(W) \geqq h^{-1} \sum_{R_{h}-C_{h}} \int_{0}^{2 h} d \xi\right]_{0}^{h} d \gamma \psi r(\xi)\left\{\left[\frac{\partial u}{\partial x}(m h+\xi, n h+\gamma)\right.\right. \\
& \left.-h^{-1}\langle W(m+1, n)-W(m, n)\rangle\right]^{2} \\
& \left.+\left[\frac{\partial u}{\partial y}(m h+\gamma, n h+\xi)-h^{-1}\langle W(m, n+) 1-W(m, n)\rangle\right]^{2}\right\} \geqq 0 .
\end{aligned}
$$

Using 2.19 and 2.21 we rewrite 2.20 as

$$
D_{R_{h}}^{(h)}(W) \leqq 3 h^{-2} \sum_{B_{h}} \iint_{S(m, n)} u^{2} d x d y+D(u),
$$

which is the desired inequality for the numerator of the Rayleigh quotient.

By definition 2.15 it is seen that for $(m, n) \cong R_{h}-C_{h}$

$$
\iint_{S(m, n)}[u(x, y)-W(m, n)] d x d y=0,
$$

so that the integrand is admissible in the variational problem for the second eigenvalue of the free membrane for the square $S(m, n)$. Consequently

$$
\frac{\pi^{2}}{h^{2}} \leqq \frac{\iint_{S(m, n)}|\operatorname{grad} u|^{2} d x d y}{\iint_{S(m, n)}[u(x, y)-W(m, n)]^{2} d x d y} .
$$

This can be written as

$$
h^{2} W(m, n)^{2} \geqq \iint_{S(m, n)} u^{2} d x d y-\frac{h^{2}}{\pi^{2}} \iint_{S(m, n)}|\operatorname{grad} u|^{2} d x d y .
$$

Then 


$$
\begin{aligned}
h^{2} \sum_{R_{h}-C_{h}} W^{2} & \geqq \sum_{R_{h}-\sigma_{h}} \iint_{S(m, n)}\left[u^{2}-\frac{h^{2}}{\pi^{2}}|\operatorname{grad} u|^{2}\right] d x d y, \\
& \geqq \iint_{R} u^{2} d x d y-\iint_{S} u^{2} d x d y-\frac{h^{2}}{\pi^{2}} D(u),
\end{aligned}
$$

where $S$ is a strip of depth $\alpha h$ inside $R$ with outer boundary $C$ which completely covers the sets $S(m, n) \cap R$, for $(m, n) \in C_{h}+B_{h}$.

The parameter $\alpha$ which appears in the strip width, $\alpha h$, depends to some extent on the region $R$ and the manner in which the mesh is placed on $R$. In Appendix $\mathrm{B}$ it is shown that

$$
a \leqq \frac{1}{2} \sqrt{34}
$$

under the hypotheses of the next section. Substituting 2.22 and 2.26 into 2.17 we have the desired inequality

$$
\begin{aligned}
\mu_{k}(h) & \leqq \max _{a_{1} \cdots a_{k}} \frac{D(u)+3 h^{-2} \iint_{R} u^{2} d x d y}{\int_{R} u^{2} d x d y-\frac{h^{2}}{\pi^{2}} D(u)-\iint_{S} u^{2} d x d y}, \\
& \leqq \frac{\lambda_{k}+3 h^{-2} \max _{a_{1} \cdots a_{k}} \iint_{S} u^{2} d x d y}{1-\frac{h^{2}}{\pi^{2}} \lambda_{k}-\max _{a_{1} \cdots a_{k}} \iint_{S} u^{2} d x d y} .
\end{aligned}
$$

Inequality 2.27 gives an explicit bound for $\lambda_{k}$ in terms of $\mu_{k}(h)$ if one has a bound for

$$
\iint_{S} u^{2} d x d y
$$

The above lower bound without the strip integrals was obtained by Weinberger [67] for a finite difference problem on a set of mesh points which includes $R_{h}$. The admissible functions in his problem vanish at points outside of this set. The bound for $\lambda_{k}-\mu_{k}$, given by 2.27 , is $O(h)$ as we shall show in the next paragraph through an explicit bound of order $h^{3}$ for the strip integral 2.28 .

4. The strip integral. For simplicity of presentation we assume $C$ to be a smooth arc whose curvature, $K$, is differentiable. Restrict $h$ to be so small that the center of curvature corresponding to any point $p$ on $C$ does not lie in $S$ near $p$; i.e., choose $h$ so that $\alpha h<\min _{o} K^{-1}$. Let the parametric representation of $C$ be

$$
C: x_{i}=g_{i}(s)
$$$$
i=1,2 \text {, }
$$ 
where $x_{1}=x, x_{2}=y$ and $s$ is the arc length along $C$. Inside the strip $S$ we make the transformation to geodesic normal coordinates $(s, n)$ in the following manner:

$$
x_{i}=g_{i}(s)+n_{i}(s) n,
$$$$
i=1,2 \cdots \text {. }
$$

Here $\left(n_{1}, n_{2}\right)$ is taken to be the unit inward normal on $C$. The Jacobian of the transformation is

$$
\frac{\partial\left(x_{1}, x_{2}\right)}{\partial(s, n)}=(1-K(s) n),
$$

and the components of the metric tensor are

$$
a^{11}=(1-K(s) n)^{-2}, a^{12}=a^{21}=0, a^{22}=1 .
$$

The normal derivative of a function $u$ on $C$ has the property that

$$
\frac{\partial u}{\partial \nu}=a^{i j} u,{ }_{i}\left(-n_{j}\right)=-\frac{\partial u}{\partial n},
$$

and hence we shall interpret $\partial u / \partial n$ in both senses. In terms of the new coordinates we have

$$
|\operatorname{grad} u|^{2}=\left\{(1-K(s) n)^{-2}\left(\frac{\partial u}{\partial s}\right)^{2}+\left(\frac{\partial u}{\partial n}\right)^{2}\right\},
$$

and the membrane equation 2.1 takes the form

$$
\begin{aligned}
(1-K n)^{-2} \frac{\partial^{2} u}{\partial s^{2}}+\frac{\partial^{2} u}{\partial n^{2}}+ & K^{\prime} n(1-K n)^{-3} \frac{\partial u}{\partial s} \\
& -K(1-K n)^{-1} \frac{\partial u}{\partial n}+\lambda u=0 .
\end{aligned}
$$

Rewriting our strip integral in terms of the new coordinates and noting that 2.16 implies $u=0$ on $C$ leads to

$$
\begin{aligned}
& \iint_{S} u^{2} d x d y=\int_{0}^{L} d s \int_{0}^{\alpha h} u^{2}(s, n)(1-K(s) n) d n \\
& =\int_{0}^{L} d s \int_{0}^{\alpha n}\left\{u(s, 0)+\frac{\partial u}{\partial n}(s, 0) n+\int_{0}^{n}(n-\xi) \frac{\partial^{2} u}{\partial \xi^{2}}(s, \xi) d \xi\right\}^{2}(1-K(s) n) d n, \\
& \leqq 2 \int_{0}^{L} d s \int_{0}^{\alpha n} n^{2}\left[\frac{\partial u}{\partial n}(s, 0)\right]^{2}(1-K(s) n) d n \\
& +2 \int_{0}^{L} d s \int_{0}^{\alpha n}\left[\int_{0}^{n}(n-\xi) \frac{\partial^{2} u}{\partial \xi^{2}} d \xi\right]^{2}(1-K(s) n) d n, \\
& \leqq \frac{2}{3}(\alpha h)^{3}(1+\alpha h K(-)) \int_{o}\left(\frac{\partial u}{\partial \nu}\right)^{2} d s \\
& +\frac{(\alpha h)^{4}}{6}\left[\frac{1+\alpha h K(-)}{1-\alpha h K(+)}\right] \iint_{S}\left(\frac{\partial^{2} u}{\partial n^{2}}\right)(1-K(s) n) d s d n \text {. }
\end{aligned}
$$


where we have used the notation

$$
\begin{aligned}
& K(-)=\text { absolute value of maximum negative curvature } \\
& K(+)=\text { absolute value of maximum positive curvature. }
\end{aligned}
$$

The last lines of 2.35 display the desired $0\left(h^{3}\right)$ character of the strip integral.

We now seek explicit bounds for the integrals appearing on the right side of 2.35. It is true that

$$
\left(\frac{\partial^{2} u}{\partial n^{2}}\right)^{2} \leqq\left(\frac{\partial^{2} u}{\partial x^{2}}\right)^{2}+2\left(\frac{\partial^{2} u}{\partial x d y}\right)^{2}+\left(\frac{\partial^{2} u}{\partial y^{2}}\right)^{2},
$$

where the right side is recognized to be the second order differential invariant. An application of the divergence theorem to this invariant, pointed out by Payne [41], is very useful here. Integrating 2.37 over $S$ gives

$$
\begin{aligned}
& \iint_{S}\left(\frac{\partial^{2} u}{\partial n^{2}}\right)^{2} d x d y \leqq \iint_{R}\left[\left(\frac{\partial^{2} u}{\partial x^{2}}\right)^{2}+2\left(\frac{\partial^{2} u}{\partial x \partial y}\right)^{2}+\left(\frac{\partial^{2} u}{\partial y^{2}}\right)^{2}\right] d x d y \\
& =D\left(u_{x}\right)+D\left(u_{y}\right)=-\iint_{R}\left[u_{x} \Delta u_{x}+u_{y} \Delta u_{y}\right] d x d y+\int_{\sigma}\left(u_{x} \frac{\partial u_{x}}{\partial \nu}+u_{y} \frac{\partial u_{y}}{\partial \nu}\right) d s .
\end{aligned}
$$

In view of 2.16 and the fact that $\partial u_{i} / \partial x, \partial u_{i} / \partial y$ each satisfy the membrane equation, 2.38 can be continued to

$$
\begin{gathered}
\iint_{S}\left(\frac{\partial^{2} u}{\partial n^{2}}\right)^{2} d x d y \\
\leqq \iint_{R}\left\{[ \sum _ { 1 } ^ { k } a _ { i } \frac { \partial u _ { i } } { \partial x } ] \left[\sum_{1}^{k} a_{i} \lambda_{i}\left(\frac{\partial u_{i}}{\partial x}\right)\right.\right. \\
\left.+\left[\sum_{1}^{k} a_{i} \frac{\partial u_{i}}{\partial y}\right]\left[\sum_{1}^{k} a_{i} \lambda_{i} \frac{\partial u_{i}}{\partial y}\right]\right\} d x d y+\frac{1}{2} \int_{\sigma} \frac{\partial}{\partial \nu}|\operatorname{grad} u|^{2} d s, \\
\leqq \sum_{1}^{k} a_{i}^{2} \lambda_{i}^{2}-\frac{1}{2} \int_{\sigma}\left\{\lim _{n \rightarrow 0} \frac{\partial}{\partial n}\left[(1-K n)^{-2}\left(\frac{\partial u}{\partial s}\right)^{2}+\left(\frac{\partial u}{\partial n}\right)^{2}\right]\right\} d s .
\end{gathered}
$$

The latter inequality follows from 2.33 and the orthogonality of the eigenfunctions is Dirichlet norm, i.e.,

$$
D\left(u_{i}, u_{j}\right)=\delta_{i j} \lambda_{(i)},
$$

Continuing the inequality we have

$$
\iint_{S}\left(\frac{\partial^{2} u}{\partial n^{2}}\right)^{2} d x d y \leqq \lambda_{k}^{2}-\int_{o}\left(\frac{\partial u}{\partial n}\right)\left(\frac{\partial^{2} u}{\partial n^{2}}\right) d s .
$$

If we now assume that the differential equation 2.34 is satisfied on the boundary in the limiting sense 2.41 becomes 


$$
\iint_{S}\left(\frac{\partial^{2} u}{\partial n^{2}}\right)^{2} d x d y \leqq \lambda_{k}^{2}+K(-) \int_{\sigma}\left(\frac{\partial u}{\partial n}\right)^{2} d s .
$$

Estimates of the contour integral above can be made from an integral identity due to Payne and Weinberger [45]. This formula which was obtained for hyperbolic operators by L. Hörmander [27] is a generalization of an integral identity of Rellich [52]. Using the summation convention the identity in two dimensions becomes

$$
\begin{aligned}
\int_{\sigma}\left\{f^{j} \nu_{j}\right. & {\left.\left[\left(\frac{\partial u}{\partial s}\right)^{2}-\left(\frac{\partial u}{\partial \nu}\right)^{2}\right]-2 f^{j} t_{j}\left(\frac{\partial u}{\partial s}\right)\left(\frac{\partial u}{\partial \nu}\right)\right\} d s } \\
& =-2 \iint_{R} f^{i} u_{, i} \Delta u d x d y+\iint_{R}\left[f^{j}{ }_{j} \delta^{i p}-2 f_{, p}^{i}\right] u_{, i} u_{, p} d x d y .
\end{aligned}
$$

In this formula $\left(t_{1}, t_{2}\right)$ is the unit tangent vector to the curve $C$ while $\left(f^{1}, f^{2}\right)$ is an arbitrary piecewise continuously differentiable vector field defined on the closure of $R$. Since $u=0$ on $C, 2.43$ reduces to

$$
\begin{aligned}
\iint_{C}\left(f^{i} \nu_{i}\right)\left(\frac{\partial u}{\partial n}\right) d s & =2 \iint_{R} f^{i} u_{, i} \Delta u d x d y \\
& -\iint_{R}\left[f_{, j}^{j} \delta^{i p}-2 f_{, j}^{i}\right] u_{, i} u_{, p} d x d y .
\end{aligned}
$$

Let us further assume that $f^{i} \nu_{i}>0$ on $C$ so that by Schwarz's inequality

$$
\begin{gathered}
\int_{o}\left(\frac{\partial u}{\partial n}\right)^{2} d s \\
\leqq \frac{1}{\min _{\sigma} f^{i} \nu_{i}}\left\{2\left[\max _{R} \sqrt{f^{i} f^{i}}\right]\left[\iint_{R}(\Delta u)^{2} d x d y\right]^{1 / 2} D(u)^{1 / 2}+D(u) \tau\right\} .
\end{gathered}
$$

Here we have used the notation

$$
\begin{aligned}
\tau & =\max _{R}\left|\left(f_{, 1}^{1}-f_{, 2}^{2}\right)^{2}+\left(f_{, 2}^{1}+f_{,,}^{2}\right)^{2}\right|^{1 / 2} \\
& =\max _{R}\left|f_{{ }^{\prime}}^{i} f_{, i}^{j}+f_{, j}^{i} f_{, j}^{i}-\left(f_{, i}^{i}\right)^{2}\right|^{1 / 2},
\end{aligned}
$$

which arises from the largest eigenvalue of the matrix

$$
\left(f_{, j}^{i}+f_{, i}^{j}-\delta_{j}^{i} f_{, k}^{k}\right) \text {. }
$$

From the definition 2.16 of $u$ the inequality 2.45 gives rise to the result

$$
\int_{o}\left(\frac{\partial u}{\partial n}\right)^{2} d s \leqq \frac{\lambda_{k}}{\min _{\sigma} f^{i} \nu_{i}}\left\{2 \sqrt{\lambda_{k}} \max _{R} \sqrt{f^{i} f^{i}}+\tau\right\} .
$$

The vector field $\left(f^{1}, f^{2}\right)$ can be defined in many ways depending upon the region $R$. One possible method is described in detail in Appendix A. If $R$ is star-shaped with respect to the origin, i.e., if every ray from 
the origin cuts $C$ in one point, we can define $f^{i}=x^{i}$. In this case 2.48 takes the form

$$
\int_{\sigma}\left(\frac{\partial u}{\partial n}\right)^{2} d s \leqq 2 \lambda_{k}^{3 / 2} \frac{\max _{R} \sqrt{x^{i} x^{i}}}{\min _{\sigma} x^{i} \nu_{i}} .
$$

Finally, upon substituting 2.42 and 2.48 into 2.35 , our original inequality 2.27 takes the form

$$
\mu_{k}(h) \leqq \frac{\lambda_{k}\left(1+h B_{k}\right)}{\left(1-h^{2} A_{k}\right)} .
$$

Here $A_{k}$ and $B_{k}$ are given by

$$
\text { (2.51) } \begin{aligned}
& A_{k}=\frac{\bar{\lambda}_{k}}{\pi^{2}} \\
+ & h \bar{\lambda}_{k}\left\{\frac{\alpha^{3}(1+\alpha h K(-)}{3 \min f^{i} \nu_{i}}\right\}\left\{2+\frac{\alpha h K(-)}{2(1-\alpha h K(+))}\right\}\left\{2 \sqrt{\bar{\lambda}_{k}} \max \sqrt{f^{i} f^{i}}+\tau\right\} \\
+ & h^{2} \bar{\lambda}_{k}^{2} \frac{\alpha^{4}}{6}\left(\frac{1+\alpha h K(-)}{1-\alpha h K(+)}\right), \\
& B_{k}=\frac{\alpha^{3}(1+\alpha h K(-)}{\min _{\sigma} f^{i} \nu_{i}}\left\{2+\frac{\alpha h K(-)}{2(1-\alpha h K(+))}\right\}\left\{2 \sqrt{\bar{\lambda}_{k}} \max _{R} \sqrt{f^{i} f^{i}}+\tau\right\} \\
+ & \frac{h \bar{\lambda}_{k} \alpha^{4}}{2}\left(\frac{1+\alpha h K(-)}{1-\alpha h K(+)}\right),
\end{aligned}
$$

and $\bar{\lambda}_{k}$ is an upper bound for $\lambda_{k}$. In particular we can use the upper bound 2.14 as $\bar{\lambda}_{k}$. Solving 2.50 yields the lower bound

$$
\lambda_{k} \geqq \frac{\mu_{k}(h)\left(1-h^{2} A_{k}\right)}{1+h B_{k}} .
$$

It is seen that the difference between the upper and lower bounds is the expression

$$
\mu_{k}(h)\left\{\left[1-\frac{h^{2}}{4} \mu_{k}(h)\right]^{-1}-\left[1-h^{2} A_{k}\right]\left[1+h B_{k}\right]^{-1}\right\},
$$

which is indeed $O(h)$ in terms of easily computed quantities. These are $\mu_{k}(h), K(-), K(+)$, and the vector field $\left(f^{1}, f^{2}\right)$ which in most instances will strongly reflect the geometry of $C$.

We note that by our choosing the mesh width $h$ to be sufficiently small the inequality 2.26 will yield

$$
h^{2} \sum_{R_{h}-\sigma_{h}} W^{2}>0 \text {. }
$$

We assume $h$ to be so chosen. This in turn assures the linear inde- 
pendence of $W_{1}, \cdots, W_{k}$ which was assumed earlier.

At the beginning of this section we had restricted $C$ to be a smooth arc for reasons of simplicity. However, discontinuities in the derivatives of the boundary $C$ offer no essential difficulty in carrying through the previous development. As before we assume that the interior angles at corners are less than $180^{\circ}$. Difficulties arise at corners because of possible bad behavior of various derivatives of $u$. This could invalidate the inequalities 2.38, 2.39 and 2.41 .

We construct the sequence of regions $R_{1}, R_{2}, \cdots$ with the properties:

(1) $R_{1} \subseteq R_{2} \subseteq \cdots \sqsubseteq R$

(2) $x \in R \Rightarrow x \in R_{i}$ for some $i$

(3) the boundary $C_{i}$ of $R_{i}$ is continuously differentiable

(4) at a corner of $C$ the locus of centers of curvature of $C_{i}$ lying with the strip $S_{i}$ of depth $\alpha h$ has the property that the normal to $C_{i}$ intersects the bisector of the angle before reaching its center of curvature.

(5) the curvature of each $C_{i}$ is positive in the vicinity of a corner.

The bounds of this section apply in the case of each $R_{i}$ using the various parameters $h, \alpha,\left(f^{1}, f^{2}\right)$, which previously have been selected for $R$. At corners of $R$ the locus of centers of curvature of $R_{i}$ may enter the strip $S$. Condition (4) above, assures that we can change the upper limit of integration in 2.35 from $\alpha h$ to $K^{-1}(\mathrm{~s})$ where such penetration occurs and still cover the domain of integration. Let $S_{i}^{\eta}$ be the sector of $S_{i}$ at the corner $\eta$ such that the locus of centers of curvature of $C_{i}^{\eta}$ lies in the strip $S_{i}$. At this corner 2.35 takes the form

$$
\begin{gathered}
\iint_{s_{i}^{\eta}} u_{i}^{2} d x d y \leqq \int_{0}^{L_{i}^{\eta}} d s \int_{0}^{b_{i}(s)} u^{2}(s, n)\left(1-K_{i}(s) n\right) d n \\
\leqq \frac{2}{3}(\alpha h)^{3} \int_{\sigma_{i}^{\eta}}\left(\frac{\partial u}{\partial \nu}\right)^{2} d s+\frac{(\alpha h)^{4}}{6} \iint_{s_{i}^{\eta}}\left(\frac{\partial^{2} u}{\partial n^{2}}\right)^{2}\left(1-K_{i}(s) n\right) d s d n .
\end{gathered}
$$

Here $b_{i}(s) \leqq K_{i}^{-1}(s)$, where $\left(s, b_{i}(s)\right)$ is on the bisector of the angle at $\eta$. The bound 2.35 is then seen to be valid for $R_{i}$ where $K_{i}(+)$ is the maximum value of the curvature of $C_{i}$ at other than points of $C_{i}^{\eta}$. The inequalities following 2.35 are also seen to be valid under this interpretation of $K_{i}(+)$, and with $\lambda_{k}$ replaced by $\lambda_{k}\left(R_{i}\right)$.

We assume the mesh to be placed on $R$ so that no mesh points lie on $C$ in the vicinity of a corner. Then for some $N$ and all $i>N$, we have

$$
\mu_{k}(h)=\mu_{k}\left(h, R_{i}\right) .
$$

Hence 2.50 becomes 


$$
\mu_{k}(h) \leqq \frac{\lambda_{k}\left(R_{i}\right)\left(1+h B_{k}\left(R_{i}\right)\right)}{\left(1-h^{2} A_{k}\left(R_{i}\right)\right)} .
$$

The hypotheses of 2.55 are sufficient conditions for

$$
\lim _{i \rightarrow \infty} \lambda_{k}\left(R_{i}\right)=\lambda_{k},
$$

and also for

$$
\begin{aligned}
& \lim _{i \rightarrow \infty} B_{k}\left(R_{i}\right)=B_{k}, \\
& \lim _{i \rightarrow \infty} A_{k}\left(R_{i}\right)=A_{k},
\end{aligned}
$$

as was stated by D. M. Eidus $[16,17]$. Therefore the bound 2.50 also holds in the case of corners whose interior angles are less than $180^{\circ}$. There seems to be little hope of applying the procedures of this paper toward bounds in the case of angles greater than $180^{\circ}$ since the factor $(1+K(-) \alpha h)$ is present, and $K_{i}(-) \rightarrow \infty$.

\section{ChAPTER III}

\section{THE FREE MEMBRANE PROBLEM}

1. The finite difference problem. The free membrane problem is given by the equations

$$
\begin{aligned}
\Delta u+\lambda u=0 & \text { in } R \\
\frac{\partial u}{\partial \nu}=0 & \text { on } C
\end{aligned}
$$

with eigenvalues $0=\lambda_{1} \leqq \lambda_{2} \leqq \cdots$. The eigenfunctions are $u_{1}, u_{2}, \cdots$ with the normalization

$$
\iint_{R} u_{i}^{2} d x d y=1
$$

The approximating finite difference problem is chosen to be

$$
\begin{aligned}
\Delta_{h} V(m, n)+\mu V(m, n) & =0, & & (m, n) \in R_{h}-C_{h} \\
\nabla(\nu) V(m, n) & =0, & & (m, n) \in C_{h}
\end{aligned}
$$

where $\nabla(\nu) V(m, n)=0$ is the condition that $V(m, n)$ be the average of the values of $V$ at adjacent (vertical and horizontal) points of $R_{h}$ only. For example in Fig. 2 the point $(m, n)$ belongs to $C_{h}$ so that

$$
\begin{aligned}
0= & \nabla(\nu) V(m, n)=V(m, n+1)+V(m+1, n) \\
& +V(m, n-1)-3 V(m, n) .
\end{aligned}
$$


Now define $C_{h}$ to be the set of grid points which have an associated triangle intersecting the boundary $C . R_{h}$ is the set of mesh points interior to $R$ augmented by points of $C_{h}$. The set $B_{h}$ is defined to be those points in $R_{h}-C_{h}$ which have an associated triangle with a vertex in $C_{h}$. See Fig. 1 .

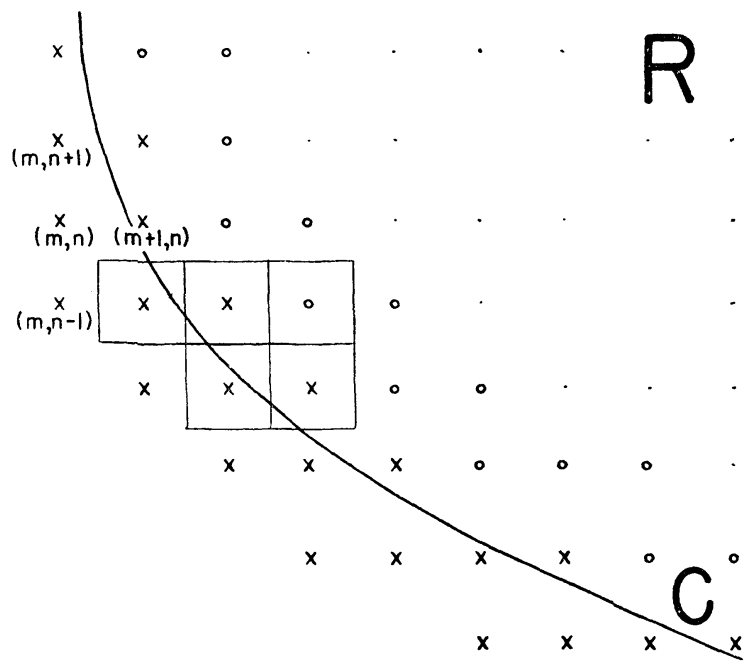

Fig. 2. Symbol Set to which point belongs

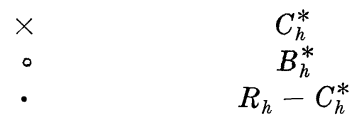

Let the eigenvalues of 3.3 be $\mu_{1}(h) \leqq \mu_{2}(h) \leqq \cdots$ with corresponding mesh eigenfunctions $V_{1}, V_{2}, \cdots$ which have the normalization

$$
h^{2} \sum_{R_{h}-C_{h}} V_{i}^{2}=1 \text {. }
$$

The minimum-maximum principle 2.8 yields the eigenvalues of the freemembrane problem 3.1 where in this case the functions involved need not vanish on the boundary $C$ to be admissible. In like manner the variational principle 2.6 gives the eigenvalues of our finite difference analogue 3.3 where any set of $k$ linearly independent mesh functions is allowed. The procedure followed to obtain bounds for $\lambda_{k}$ in terms of $\mu_{k}(h)$ is very similar to that of Chapter II for the fixed membrane problem.

To obtain an upper bound for $\lambda_{k}$ we define a set of functions $v_{1}, \cdots$, $v_{k}$ which are admissible in the variational problem 2.8 in terms of the mesh eigenfunctions $V_{1}, \cdots, V_{k}$. Let $v_{j}(x, y)$ be the piecewise continuously differentiable function which is linear in each triangle and which coincides at the vertices with $V_{j}(m, n)$. By considerations identical to those of Chapter II, §2, we arrive at the upper bound 


$$
\lambda_{k} \leqq \frac{\mu_{k}(h)}{1-\frac{h^{2}}{4} \mu_{k}(h)} .
$$

A lower bound for $\lambda_{k}$ is achieved by finding an appropriate upper bound for $\mu_{k}(h)$ in terms of $\lambda_{k}$. As before $S(m, n)$ is defined to be the "associated" square with $(m h, n h)$ as center and sides of length $h$ having the same orientation as the grid lines. We can achieve somewhat better bounds by altering the set $C_{h}$ slightly. Define $C_{h}^{*}$ to be those points of $C_{h}$ whose associated squares intersect the boundary. Let $B_{h}^{*}$ be the set of interior mesh points of $R$ whose squares share a common vertex with at least one of the squares of $C_{h}^{*}$, e.g. see Fig. 2. We define a linearly independent set of $k$ mesh functions $W_{1}, \cdots, W_{k}$ in terms of the eigenfunctions $u_{1}, \cdots, u_{k}$ as follows:

$$
\begin{aligned}
W_{i}(m, n)=h^{-2} \iint_{S(m, n)} u_{i}(x, y) d x d y, & (m, n) \in R_{h}-C_{h}^{*}, \\
\nabla(\nu) W_{\imath}(m, n)=0, & (m, n) \in C_{h}^{*} .
\end{aligned}
$$

Note that $\nabla(\nu) W_{i}=0$ represents as many linear equations as unknowns. We further define

$$
\begin{aligned}
W & =a_{1} W_{1}+\cdots+a_{k} W_{k}, \\
u & =a_{1} u_{1}+\cdots+a_{k} u_{k} .
\end{aligned}
$$

By the same considerations used in proving 2.21 we see that

$$
D_{R_{h}-\sigma_{h}^{*}}^{(h)}(W) \leqq D(u) \text {. }
$$

The above sum is taken over all differences of neighboring points of $R_{h}-C_{h}^{*}$. It follows immediately that

$$
D_{R_{h}}^{(h)}(W) \leqq D(u)+\beta(W) D_{B_{h}^{\prime}}^{(h)}(W),
$$

where

$$
\beta(W)=\frac{D_{R_{h}}^{(h)}(W)-D_{R_{h}-C_{h}^{*}}^{(h)}(W)}{D_{B_{h}^{*}}^{(h)}(W)} .
$$

The quantity $\beta(W)$ is minimized with respect to possible choices of $W$ at points of $C_{h}^{*}$ by the manner in which the functions $W_{1}, \cdots, W_{k}$ were defined at these points. In fact if $(m, n) \in C_{h}^{*}$ as in Fig. 2 we see that

$$
\begin{gathered}
\frac{d}{d W(m, n)} \beta(W)=2\{3 W(m, n)-W(m, n+1)-W(m+1, n) \\
-W(m, n-1)\} D_{B_{h}^{*}}^{(h)}(W)^{-1} \\
=2 \nabla(\nu) W(m, n) D_{B_{h}^{*}}^{(h)}(W)^{-1}=0
\end{gathered}
$$


which is the desired result. The bound for $\beta(W)$ depends to some extent on the geometry of $C$ and consequently must be determined anew for each region $R$. Assume that $h$ is chosen small enough so that the points of $C_{h}^{*}$ constitute at most a double "fence" around $B_{h}^{*}$. In such a case we can choose the values of $W_{i}$ on $C_{h}^{*}$ in terms of nearby points of $B_{h}^{*}$ so that

$$
\beta(W) \leqq 4
$$

For a further discussion of $\beta$ see Appendix B.

Finally we can write 3.8 in the form

$$
D_{R_{h}}^{(h)}(W) \leqq D(u)+\beta(W) \iint_{S}|\operatorname{grad} u|^{2} d x d y,
$$

where again $S$ is a strip of suitable depth, $\bar{\alpha} h$, so that the squares associated with points of $B_{h}^{*}$ lie in $S$. In Appendix B the parameter $\bar{\alpha}$ is discussed and it is shown that, under the hypotheses of this section, we can take

$$
\bar{\alpha} \leqq 2 \sqrt{2}
$$

We observe, in addition, that 2.26 is valid for this same strip $S$ so that we can write the lower bound in the form

$$
\mu_{k}(h) \leqq \frac{\lambda_{k}+\bar{\beta} \max _{a_{1} \cdots a_{k}} \iint_{S}|\operatorname{gradu}|^{2} d x d y}{1-\frac{h^{2}}{\pi^{2}} \lambda_{k}-\max _{a_{1} \cdots a_{k}} \iint_{S} u^{2} d x d y}
$$

where $\bar{\beta}$ is a numerical bound for $\beta(W)$, i.e., $\beta(W) \leqq \bar{\beta}$.

2. Bounds for the strip integrals. We are faced with the task of bounding each of two integrals in the free membrane problem over a strip of depth $\bar{\alpha} h$ adjacent to the boundary $C$. We desire the bounds themselves to be of order $h$. As in Chapter II, $\S 4$, we assume $C$ to be a smooth arc whose curvature $K$ is differentiable. Restrict $h$ to be so small that the center of curvature corresponding to any point $p$ on $C$ does not lie in $S$ near $p$; i.e., choose $h$ so that $\alpha h<\min _{0} K^{-1}$. The geodesic normal coordinates $(s, n)$ are introduced as before so that equations 2.29 through 2.34 remain valid. We see from 2.30 that this guarantees the single-valuedness of our transformation. An additional identity involving the second order differential invariant will be useful in the estimates which follow, and is included at this time. If $\left.u\right|_{i j}$ denotes covariant differentiation then 
(3.14)

$$
\begin{aligned}
u_{x x}^{2} & +2 u_{x y}^{2}+u_{y y}^{2}=a^{i j} a^{p m} u_{\mid i p} u_{\mid j m}=\left(a^{11} u_{\mid 11}\right)^{2}+2 a^{11} a^{22}\left(u_{\mid 12}\right)^{2} \\
& +\left(a^{22} u_{\mid 22}\right)^{2}=\left\{( 1 - K n ) ^ { - 2 } \left[\frac{\partial^{2} u}{\partial s^{2}}+\frac{K^{\prime} n}{(1-K n)} \frac{\partial u}{\partial s}\right.\right. \\
& \left.\left.-K(1-K n) \frac{\partial u}{\partial n}\right]\right\}^{2}+2(1-K n)^{-2}\left[\frac{\partial^{2} u}{\partial s \partial n}+\frac{K}{(1-K n)} \frac{\partial u}{\partial s}\right]^{2} \\
& +\left(\frac{\partial^{2} u}{\partial n^{2}}\right)^{2}
\end{aligned}
$$

The strip integral appearing in the numerator of 3.13 can be bounded using 2.33 and 3.14 as follows:

$$
\begin{aligned}
& \iint_{s}|\operatorname{grad} u|^{2} d x d y=\int_{0}^{L} \int_{0}^{\bar{\alpha} h}\left\{(1-K n)^{-2}\left(\frac{\partial u}{\partial s}\right)^{2}+\left(\frac{\partial u}{\partial n}\right)^{2}\right\}(1-K n) d n d s \\
& =\int_{0}^{L} \int_{0}^{\bar{\alpha} h}\left\{\left[\frac{\partial u}{\partial s}(s, 0)+\int_{0}^{n} \frac{\partial}{\partial t}\left\langle(1-K t)^{-1} \frac{\partial u}{\partial s}(s, t)\right\rangle d t\right]^{2}\right. \\
& \left.+\left[\int_{0}^{n} \frac{\partial^{2} u}{\partial t^{2}}(s, t) d t\right]^{2}\right\}(1-K n) d n d s \\
& \quad \leqq \int_{0}^{L} \int_{0}^{\bar{\alpha} h}\left\{2\left[\frac{\partial u}{\partial s}(s, 0)\right]^{2}\right. \\
& +n \int_{0}^{n}\left\langle(1-K t)^{-2}\left[\frac{\partial^{2} u}{\partial s \partial t}(s, t)+\frac{K}{(1-K t)} \frac{\partial u}{\partial s}(s, t)\right]^{2}\right. \\
& \left.\left.+\left[\frac{\partial^{2} u(s, t)}{\partial t^{2}}\right]^{2}\right\rangle d t\right\}(1-K n) d n d s \\
& \leqq 2(\bar{\alpha} h)(1+\bar{\alpha} h K(-)) \int_{o}\left(\frac{\partial u}{\partial s}\right)^{2} d s \\
& +\frac{(\bar{\alpha} h)^{2}}{2}\left(\frac{1+\bar{\alpha} h K(-)}{1-\bar{\alpha} h K(+)}\right) \iint_{s} a^{i j} a^{p m} u_{\mid i p} u_{\mid j m} d x d y .
\end{aligned}
$$

The integral over the strip $S$ of the second order differential invariant can be bounded as follows:

$$
\begin{gathered}
\left.\left.\iint_{S} a^{i j} a^{p m} u\right|_{i p} u\right|_{j m} d x d y=D\left(u_{x}\right)+D\left(u_{y}\right) \\
\leqq-\iint_{R}\left[u_{x} \Delta u_{x}+u_{y} \Delta u_{y}\right] d x d y+\frac{1}{2} \int_{o} \frac{\partial}{\partial \nu}|\operatorname{grad} u|^{2} d s \\
\leqq \sum_{1}^{k} a_{i}^{2} \lambda_{i}^{2}-\frac{1}{2} \int_{o} \lim _{n \rightarrow 0} \frac{\partial}{\partial n}\left[(1-K n)^{-2}\left(\frac{\partial u}{\partial s}\right)^{2}\right] d s \\
\leqq \lambda_{k}^{2}+K(-) \int_{o}\left(\frac{\partial u}{\partial s}\right)^{2} d s .
\end{gathered}
$$

Using this bound, the inequality 3.15 can be expressed in the form 


$$
\begin{gathered}
\iint_{s}|\operatorname{grad} u|^{2} d x d y \leqq \frac{\lambda_{k}^{2}(\bar{\alpha} h)^{2}}{2}\left(\frac{1+\bar{\alpha} h K(-)}{1-\bar{\alpha} h K(+)}\right) \\
+(2 \bar{\alpha} h)(1+\bar{\alpha} h K(-))\left[1+\left(\frac{\bar{\alpha} h}{2}\right)^{2} \frac{K(-)}{1-\bar{\alpha} h K(+)}\right] \int_{o}\left(\frac{\partial u}{\partial s}\right)^{2} d s .
\end{gathered}
$$

Given a piecewise continuously differentiable vector field $\left(f^{1}, f^{2}\right)$ defined in $R$ and on $C$ we can apply the integral identity 2.43 due to Payne and Weinberger to our function $u$ defined by 3.7 to yield

$$
\begin{gathered}
\int_{0} f^{k} \nu_{k}\left(\frac{\partial u}{\partial s}\right)^{2} d s=-2 \iint_{R} f^{i} u_{, i} \Delta u d x d y \\
+\iint_{R}\left[f_{, k}^{k} \delta^{i j}-2 f_{, j}^{i}\right] u_{, i} u_{, j} d x d y .
\end{gathered}
$$

If we impose the further condition that $f^{i} \nu_{i}>0$ on $C$, equation 3.18 yields the inequality

$$
\int_{o}\left(\frac{\partial u}{\partial s}\right)^{2} d s \leqq \frac{\lambda_{k}}{\min _{\sigma} f^{i} \nu_{i}}\left\{2 \sqrt{\lambda_{k}} \max _{R} \sqrt{f^{i} f^{i}}+\tau\right\},
$$

where $\tau$ is given by 2.44 . If $R$ is star shaped with respect to the origin we can let $f^{i}=x^{i}$ and 3.19 takes the form

$$
\int_{\sigma}\left(\frac{\partial u}{\partial s}\right)^{2} d s \leqq 2 \lambda_{k}^{3 / 2}\left[\frac{\max _{R} \sqrt{x^{i} x^{i}}}{\min _{\sigma} x^{i} \nu^{i}}\right] .
$$

Methods for prescribing the vector field $\left(f^{1}, f^{2}\right)$ for more general regions are discussed in Appendix A.

We now return to the estimation of the strip integral appearing in the denominator of 3.13 .

$$
\begin{aligned}
& \text { (3.21) } \iint_{s} u^{2} d x d y=\int_{0}^{L} \int_{0}^{\bar{\alpha} h}\left[u(s, 0)+\int_{0}^{n} \frac{\partial u}{\partial t}(s, t) d t\right]^{2}(1-K n) d n d s \\
& \leqq 2(\bar{\alpha} h)(1+\bar{\alpha} h K(-)) \int_{\sigma} u^{2} d s+(\bar{\alpha} h)^{2}\left(\frac{1+\bar{\alpha} h K(-)}{1-\bar{\alpha} h K(+)}\right) \iint_{s}|\operatorname{grad} u|^{2} d x d y .
\end{aligned}
$$

An application of the divergence theorem, used in a similar manner by Payne and Weinberger (45), gives

$$
\int_{O} g^{j} \nu_{j} u^{2} d s=\iint_{R} u^{2} g^{j},{ }_{j} d x d y+2 \iint_{R} u u{ }_{, j} g^{j} d x d y,
$$

where $\left(g^{1}, g^{2}\right)$ is again a piecewise continuously differentiable vector field. Assuming further that $g^{j} \nu_{j}>0$ on $C$ we have 
$(3.23)$

$$
\begin{aligned}
& \int_{\sigma} u^{2} d s \leqq \frac{1}{\min _{\sigma} g^{j} \nu_{j}}\left\{\max _{R}\left(g_{, j}^{i}\right) \iint_{R} u^{2} d x d y\right. \\
& \left.+2 \max _{R} \sqrt{g^{j} g^{j}} \sqrt{\iint_{R} u^{2} d x d y} \sqrt{D(u)}\right\} \\
& \leqq \frac{1}{\min _{\sigma} g^{j} \nu_{j}}\left[\max _{R} g_{{ }_{j}^{j}}+2 \sqrt{\lambda_{k}} \max _{R} \sqrt{g^{j} g^{j}}\right] .
\end{aligned}
$$

For star shaped regions we let $g^{j}=x^{j}$ and this inequality becomes

$$
\int_{o} u^{2} d s \leqq \frac{2}{\min _{\sigma} g^{j} \nu_{j}}\left[1+\sqrt{\lambda_{k}} \max _{R} \sqrt{x^{j} x^{j}}\right] .
$$

Substituting 3.23 into 3.21 yields the inequality

$$
\begin{aligned}
& \iint_{S} u^{2} d x d y \leqq \frac{2 \bar{\alpha} h(1+\bar{\alpha} h K(-)}{\min _{C} g^{j} \nu_{j}}\left[\max _{R} g_{, j}^{j}+2 \sqrt{\lambda_{k}} \max _{R} \sqrt{g^{j} g^{j}}\right] \\
& +(\bar{\alpha} h)^{2} \lambda_{k}\left(\frac{1+\bar{\alpha} h K(-)}{1-\bar{\alpha} h K(+)}\right),
\end{aligned}
$$

which has the desired $O(h)$ property.

By using 3.17, 3.19 and 3.25 in the inequality 3.13 we arrive at the result

$$
\mu_{k}(h) \leqq \frac{\lambda_{k}\left(1+h B_{k}\right)}{\left(1-h A_{k}\right)}
$$

where

$$
\begin{aligned}
A_{k}=\frac{2 \bar{\alpha}(1+\bar{\alpha} h K(-)}{\min _{c} g^{j} \nu_{j}}\left[\max _{R} g_{, j}^{j}+2 \sqrt{\bar{\lambda}_{k}} \max _{R} \sqrt{g^{j} g^{j}}\right] \\
+\bar{\alpha}^{2} h \bar{\lambda}_{k}\left(\frac{1+\bar{\alpha} h K(-)}{1-\bar{\alpha} h K(+)}\right)+\frac{h \bar{\lambda}_{k}}{\pi^{2}}, \\
B_{k}=\frac{2 \bar{\alpha} \bar{\beta}(1+\bar{\alpha} h K(-))}{\min _{o} f^{i} \nu_{i}}\left[1+\left(\frac{\bar{\alpha} h}{2}\right)^{2} \frac{K(-)}{(1-\bar{\alpha} h K(+))}\right] \\
\cdot\left[2 \sqrt{\bar{\lambda}_{k}} \max _{R} \sqrt{f^{i} f^{i}}+\tau\right]+\frac{\bar{\beta} \bar{\lambda}_{k} \bar{\alpha}^{2} h}{2}\left(\frac{1+\bar{\alpha} h K(-)}{1-\bar{\alpha} h K(+)}\right)
\end{aligned}
$$

and $\bar{\lambda}_{k}$ is an upper bound for $\lambda_{k}$. In particular we can use the upper bound 3.5 for $\bar{\lambda}_{k}$. Solving 3.26 yields the lower bound

$$
\lambda_{k} \geqq \frac{\mu_{k}(h)\left(1-h A_{k}\right)}{\left(1+h B_{k}\right)} .
$$

As is the case with the fixed membrane the presence of corners on 
$C$ causes difficulties in applying the previous development directly. This is because the function $u(x, y)$ may have singular behavior at the corner.

Following the approach of Chapter II we define a sequence of regions $R_{1} \subseteq R_{2} \subseteq \cdots \leqq R$ with the properties of 2.55. The bounds given by 3.17 and 3.25 can be shown to hold for each $R_{i}$ where the parameters $\bar{\alpha}, \beta, h,\left(f^{1}, f^{2}\right),\left(g^{1}, g^{2}\right)$ are those chosen for $R$. Of course the locus of centers of curvature for $R_{i}$ may now enter the strip $S$ near a corner. Where this happens we obtain the bounds for the strip integrals at the corner by special considerations.

Condition (4) of 2.55 assures that we can change the upper limit of integration at the corners in the strip integrals appearing in 3.15 to 3.22 from $\alpha h$ to $K^{-1}(s)$. If $C^{\eta}$ and $S^{\eta}$ pertain to a typical corner, $\eta$, then at that corner 3.15 becomes

$$
\begin{gathered}
\iint_{s_{i}^{\eta}}|\operatorname{grad} u|^{2} d x d y \\
=\int_{0}^{L_{i}^{\eta}} \int_{0}^{b_{i}^{(s)}}\left\{(1-K n)^{-2}\left(\frac{\partial u}{\partial s}\right)^{2}+\left(\frac{\partial u}{\partial n}\right)^{2}\right\}(1-K n) d n d s, \\
\leqq 2(\bar{\alpha} h) \int_{\sigma_{i}^{\eta}}\left(\frac{\partial u}{\partial s}\right)^{2} d s+\left.\left.\frac{(\bar{\alpha} h)^{2}}{2} \iint_{s_{i}^{\eta}} a^{i j} a^{p m} u\right|_{i p} u\right|_{j m} d x d y .
\end{gathered}
$$

This is the same as 3.15 with the factor $(1+\bar{\alpha} h K(-))$ missing. By the same reasoning 3.21 becomes

$$
\iint_{S_{i}^{\eta}} u^{2} d x d y \leqq 2(\bar{\alpha} h) \int_{\sigma_{i}^{\eta}} u^{2} d s+(\bar{\alpha} h)^{2} \iint_{S_{i}^{\eta}}|\operatorname{grad} u|^{2} d x d y .
$$

The inequalities 3.15-3.24 are true for $R_{i}$ if we interpret $K(s)$ as $K\left(s, R_{i}\right)$, $\lambda_{k}$ as $\lambda_{k}\left(R_{i}\right)$ and

$$
K\left(+, R_{i}\right)=\max _{\sigma_{i^{-}-\sigma_{i}^{\eta}}} K\left(s, C_{i}\right) .
$$

We assume the mesh to be placed on $R$ so that no mesh points lie on $C$ in the vicinity of a corner. Then for some $N$ and $i>N$ we have

$$
\mu_{k}(h)=\mu_{k}\left(h, R_{i}\right) \text {. }
$$

Then 3.26 becomes

$$
\mu_{k}(h) \leqq \frac{\lambda_{k}\left(R_{i}\right)\left(1+h B_{k}\left(R_{i}\right)\right)}{\left(1-h A_{k}\left(R_{i}\right)\right)} .
$$

By a result of D. M. Eidus $[16,17]$ the hypotheses of 2.55 imply that

$$
\lim _{i \rightarrow \infty} \lambda_{k}\left(R_{i}\right)=\lambda_{k} \text {. }
$$




$$
\begin{aligned}
& \lim _{i \rightarrow \infty} B_{k}\left(R_{i}\right)=B_{k}, \\
& \lim _{i \rightarrow \infty} A_{k}\left(R_{i}\right)=A_{k},
\end{aligned}
$$

since $B_{k}$ and $B_{k}\left(R_{i}\right)$ differ only in the value of the argument $\lambda_{k}$. Hence the bound 3.28 holds in the case of corners with interior angle less than $180^{\circ}$.

\section{APPENDIX A \\ THE VECTOR FIELD $\left(f^{1}, f^{2}\right)$}

One manner of choosing the vector field $\left(f^{1}, f^{2}\right)$ for regions star-shaped with respect to the origin which generalizes readily to more arbitrary domains is the following:

Introduce polar coordinates $(r, \theta)$ given by

$$
\begin{aligned}
& x^{1}=r \cos \theta, \\
& x^{2}=r \sin \theta .
\end{aligned}
$$

Let the boundary $C$ be a smooth curve with the polar representation

$$
C: r=\rho(\theta) .
$$

The vector field $\left(f^{1}, f^{2}\right)$ is defined as

$$
\begin{aligned}
& f^{1}\left(x^{1}, x^{2}\right)=\frac{r}{\rho(\theta)} \nu^{1}(\theta)=\frac{r}{\rho(\theta)} t^{2}(\theta), \\
& f^{2}\left(x^{1}, x^{2}\right)=\frac{r}{\rho(\theta)} \nu^{2}(\theta)=-\frac{r}{\rho(\theta)} t^{1}(\theta) .
\end{aligned}
$$

In $4.3,\left(t^{1}, t^{2}\right)$, the unit tangent vector, is given by

$$
\begin{aligned}
& t^{1}(\theta)=\frac{d x^{1}}{d s}=\left(\frac{d x^{1}}{d \theta}\right)\left(\frac{d \theta}{d s}\right)=H^{-1} \frac{d}{d \theta}[\rho(\theta) \cos \theta], \\
& t^{2}(\theta)=H^{-1} \frac{d}{d \theta}[\rho(\theta) \sin \theta],
\end{aligned}
$$

with

$$
H=\sqrt{\rho^{2}+\dot{\rho}^{2}} .
$$

A computation shows that

$$
\begin{aligned}
& \min _{\sigma} f^{i} \nu_{i}=1, \\
& \max _{R} \sqrt{f^{i} f^{i}}=1,
\end{aligned}
$$




$$
\tau=\max _{R}\left\{\frac{1}{H^{4} \rho^{4}}\left[\rho^{2} \dot{\rho}^{2}\left(\rho^{2}+3 \dot{\rho}^{2}\right)+\rho^{3} \ddot{\rho}\left(\rho \ddot{\rho}-2 \dot{\rho}^{2}\right)+\dot{\rho}^{6}\right]\right\}^{1 / 2} .
$$

It is easily verified that $\left(f^{1}, f^{2}\right)$ is piecewise continuously differentiable since, by our assumption, $\nu^{i}(s)$ is continuously differentiable on $C$. Indeed, a discontinuity in the derivative of $\left(\nu^{1}(\theta), \nu^{2}(\theta)\right)$ at $\theta_{0}$ will be propagated as a discontinuity in the derivative of $\left(f^{1}, f^{2}\right)$ along the radius vector joining $(0,0)$ to $\left(\rho\left(\theta_{0}\right), \theta_{0}\right)$. If $\left(\nu^{1}(\theta), \nu^{2}(\theta)\right.$ is itself discontinuous at a point $\theta_{0}$, i.e., if the boundary $C$ has a corner, then the quantity $\tau$ in 4.6 does not exist. In this case we replace $\left(\nu^{1}(\theta), \nu^{2}(\theta)\right)$ in 4.3 by a continuously differentiable vector field $\left(g^{1}(\theta), g^{2}(\theta)\right)$ which has the property

$$
g^{i}(\theta) \nu_{i}(\theta)>0 .
$$

The values of the quantities

$$
f^{i} \nu_{i}, \sqrt{f^{i} f^{i}}, \tau,
$$

are then computed as functions of $\left(g^{1}, g^{2}\right)$.

If $R$ is not itself star-like we subdivide $R$ into star shaped regions $R_{1}, R_{2}, \cdots, R_{N}$ whose bounding curves are given by $C_{1}, C_{2}, \cdots, C_{N}$. Let

$$
C_{i}=C_{\imath}^{\prime} U C_{i}^{\prime \prime}, \quad i=1,2, \cdots, N \text {, }
$$

where $C_{i}^{\prime}$ is that portion of $C_{i}$ which coincides with $C$, (see Fig. 3) We define the vector field $\left(g_{i}^{1}, g_{i}^{2}\right)$ on $C_{i}$ in terms of polar coordinates $\left(R_{i}, \theta_{i}\right)$

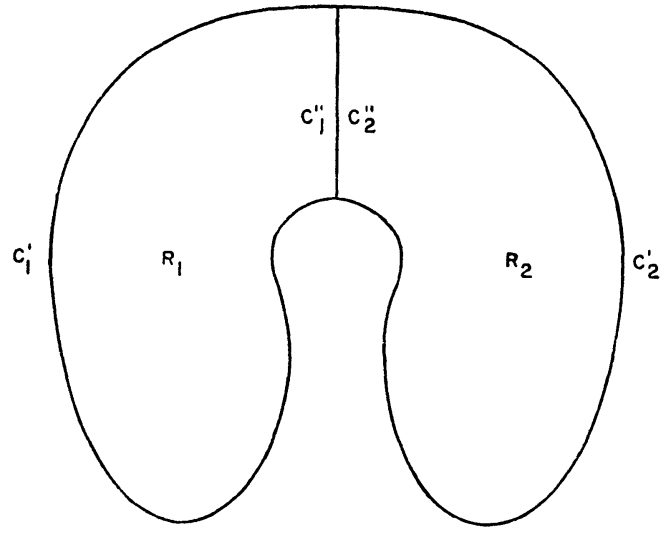

Fig. 3.

introduced in $R_{i}$. The functions $\left(g_{i}^{1}\left(\theta_{i}\right), g_{i}^{2}\left(\theta_{i}\right)\right)$ are assumed continuous on $C_{i}$ with the property

$$
g_{i}^{j}\left(\theta_{i}\right) \nu_{j}>0
$$

on $C_{i}^{\prime}$. We further assume that the functions $\left(g^{1}, g^{2}\right)$ given by 


$$
g^{j}\left(x^{1}, x^{2}\right)=g_{i}^{j}\left(\theta_{i}\right),\left(x^{1}, x^{2}\right) \in C_{i},
$$

form a piecewise continuously differentiable vector field defined on $C_{1} U C_{2} U$ $\cdots U C_{N}$. We define the vector field

$$
f^{j}\left(x^{1}, x^{2}\right)=\frac{r_{i}}{\rho_{i}\left(\theta_{i}\right)} g_{i}^{j}\left(\theta_{i}\right), \quad j=1,2,\left(x^{1}, x^{2}\right) \in R_{i},
$$

in $R$. The quantities 4.8 are then found to be

$$
\begin{gathered}
\min _{\sigma} f^{j} \nu_{j}=\min _{i=1, \cdots, N} \min _{o_{i}^{1}} f^{j_{\nu}}, \\
\max _{R} \sqrt{\overline{f^{j} f^{j}}}=\max _{i=1, \cdots, N} \max _{R_{i}} \sqrt{f^{j} f^{j}}, \\
\tau=\max _{i=1, \cdots, N} \max _{R_{i}}\left[\left(f_{, 1}^{1}-f_{, 2}^{2}\right)^{2}+\left(f_{, 2}^{1}+f_{, 1}^{2}\right)^{2}\right]^{1 / 2} .
\end{gathered}
$$

\section{APPENDIX B}

\section{THE PARAMETERS $\alpha, \bar{\alpha}$ AND $\beta$}

1. A bound for $\alpha$. For the parameter $\alpha$ we have the bound

$$
\alpha \leqq \frac{1}{2} \sqrt{34},
$$

as will now be shown under the assumptions of Chapter II, $\S 4$. We recall that the strip $S$ is the region swept out by a segment of the inward normal length $\alpha h$ as that normal moves along the curve $C$. The parameter $\alpha$ must be chosen large enough so that $S$ will cover the squares associated with the points of $B_{h}$.

In Fig. 4 we have pictured a typical case. Here the points $A, E, G$, and $H$ belong to $C_{h}$ and $B$ belongs to the set $B_{h}$. The point $F$ belonging to the square associated with $B$ is the center of the two circular arcs shown in the figure. Let $J$ be a point on $C$ with inward normal $\vec{n}$ and tangent $\vec{t}$ such that $\vec{n}$ passes through $F$. For inequality 5.1 to hold we must show that $J$ cannot lie outside the circle through $G$ about $F$ whose radius is $h / 2 \sqrt{34}$.

Assume that $J$ does lie outside the arc through $G$, as shown in the figure, with the center of curvature of $C$ at $F$. This assumption gives our boundary curve the maximum curvature at $J$ allowable under the hypotheses. As the curve $C$ continues onward it must intersect one of the triangles associated with the point $A$. At the same time the family of normals of $C$ cannot intersect within the strip $S$, i.e., the locus of centers of curvature cannot move toward $J$. The curve $C$ can most rapidly turn toward the triangle associated with $A$ if the center of curvature remains 


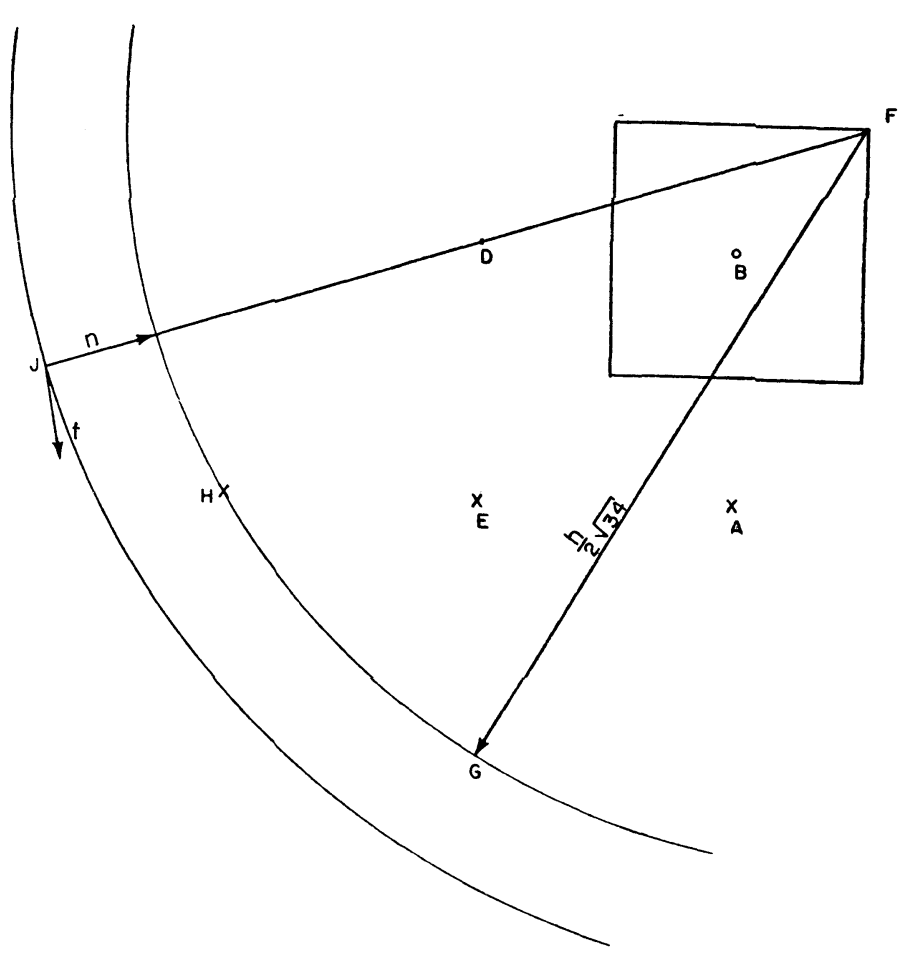

Fig. 4.

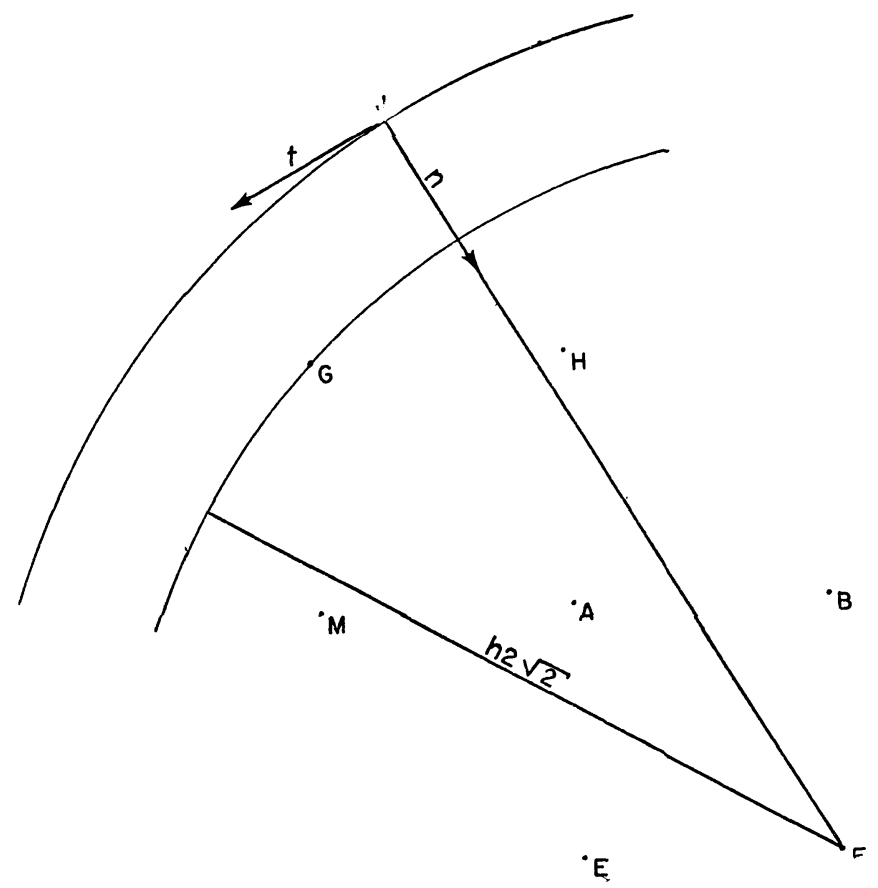

Fig. 5. 
at $F$. Clearly such a circle with center at $F$ will never intersect any traingle associated with $A$ and we have arrived at a contradiction.

2. A bound for $\bar{\alpha}$. For the parameter $\bar{\alpha}$ we have the bound

$$
\bar{\alpha} \leqq 2 \sqrt{2},
$$

which is smaller than 5.1 since the strip $S$ as defined in Chapter III $\S 1$, is somewhat narrower. In fact $S$ must cover only those squares which intersect $C$ and their neighbors in $R$ with whom they share a common side.

In Fig. 5 we have a typical situation where the square $F B A E$ shares a common vertex with $A H G M$ through which $C$ is assumed to pass. The point $F$ is taken as center of the two circles in the figure. Again we assume $J$ to be a point on $C$ whose inward normal $\vec{n}$ passes through $F$. For inequality 5.2 to hold we must show that $J$ cannot lie outside the circle through $G$ about $F$ whose radius is $2 h \sqrt{2}$.

As before we assume that $J$ does lie outside the arc through $G$, as shown in the figure, with the center of curvature of $C$ at $F$. This assumption gives our boundary curve the maximum curvature at $J$ allowed by the hypotheses. As the curve $C$ continues onward, it must intersect the square $A H G M$. The curve $C$ can most rapidly turn toward $A H G M$ under the constraints imposed if it is the circle with center at $F$. Hence the contradiction.

3. A bound for $\beta$. We shall first show that, under the assumption the points of $C_{h}^{*}$ form a double fence of the type shown in Fig. 6, an upper bound $\bar{\beta}$ for $\beta$ is

$$
\beta \leqq \bar{\beta}=4 \text {. }
$$

The actual selection of $W_{i}$ minimizes $\beta(W)$ over $C_{h}^{*}$ and hence any other assignment of values to $W_{i}$ will provide an upper bound for $\beta$. First, we shall see how to assign values to the points of Fence 2 in terms of the values at points of Fence 1 so that

$$
\underset{\text { Fence } 1+\text { Fence } 2}{D_{(h)}}-\underset{\text { Fence } 1}{D_{(h)}(V) \leqq} \underset{\text { Fence } 1}{2} D^{(h)}(V) .
$$

In fact assume that the capital letters represent the values of $V$ on Fence 1 in the figure, and consider the assignment made there at points of Fence 2. It is readily verified that property 5.4 holds for this scheme of assigning values to Fence 2. Therefore see that one can step inward from Fence $n$ one fence at a time in assigning values with the result that

$$
\underset{\substack{N \\ n=1}}{D_{\text {Fence } n}^{(h)}}(V)-\underset{\text { Fence 1 }}{D_{(h)}^{(h)}}(V) \leqq \underset{\text { Fence 1 }}{2^{N-1}} \boldsymbol{2}^{(h)}(V)
$$




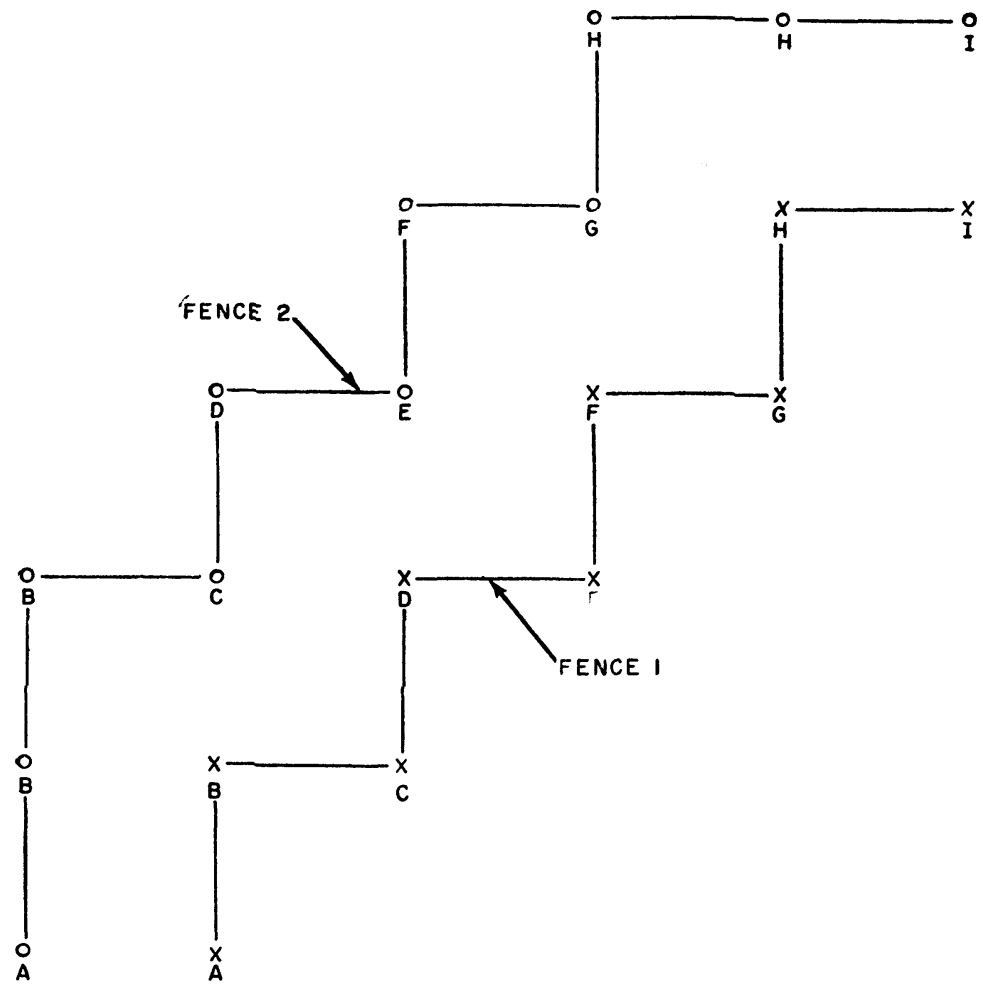

Fig. 6 .

Applying 5.5 to 3.9 with $N=3$ gives the result 5.3. The requirement on $h$ that $(1-\alpha h K)>0$ isn't sufficient to allow us to take $N=3$. In addition to a local condition of this nature a restiction "in the large" is also called for. In particular we must assure that the squares of $R_{h}-C_{h}^{*}$ form approximately the same geometrical figure as $R$. For example, if $R$ is hour-glass shaped, the squares of $R_{h}-C_{h}^{*}$ might form doubly-connected regions. If $R$ is snake-like the set $R_{h}-C_{h}^{*}$ might even be empty. Such an additional condition is as follows: No adjoining squares of $C_{h}^{*}$ may represent remote sections of the boundary $C$.

\section{APPENDIX C}

\section{SOME EXAMPLES}

In this section we seek to gain some knowledge of the relative size of the quantities involved in the bounds for the fixed membrane. Computations have been made here for the first eigenvalue of two geometric configurations; (a) the square, and (b) the unit circle. In each case $\lambda_{1}$ and $u_{1}$ are known so the strip integral 2.28 can be evaluated explicitly 
and compared with the bound obtained from $\S 2$ of Chapter II.

1. The unit square. Let $R$ be the unit square with vertices $(0,0)$, $(0,1),(1,1)$, and $(1,0)$. The first eigenvalue and eigenfuction are

$$
\begin{aligned}
& \lambda_{1}=2 \pi^{2}, \\
& u_{1}=2 \sin \pi x \sin \pi y .
\end{aligned}
$$

If we assume $h=\frac{1}{N}, N$ an integer, then we can take

$$
\alpha=\frac{3}{2}
$$

Let the vector field $\left(f^{1}, f^{2}\right)$ be chosen as

$$
\begin{aligned}
& f^{1}=x-\frac{1}{2}, \\
& f^{2}=y-\frac{1}{2},
\end{aligned}
$$

so that

$$
\begin{aligned}
\tau & =0, \\
\min _{\sigma} f^{i} \nu_{i} & =\frac{1}{2}, \\
\max _{R} \sqrt{f^{i} f^{i}} & =\frac{\sqrt{2}}{2} .
\end{aligned}
$$

A direct computation shows that in 2.51

$$
B_{1}=27 \pi+O(h) \text {. }
$$

The quantity $B_{1}$ involves bounds on the strip integral. Define $\bar{B}_{1}$ to be the analogue of $B_{1}$ with the strip integral substituted for the bound. Then

$$
\bar{B}_{1}=\frac{2 \iint_{S} u_{1}^{2} d x d y}{h^{3} \lambda_{1}}
$$

We see that in this case

$$
\bar{B}_{1}=\frac{27}{2}+O\left(h^{2}\right) \text {. }
$$

The difference between the upper and lower bounds given by 2.53 be- 
comes

$$
\mu_{1}(h) 27 \pi h+O\left(h^{2}\right) \text {. }
$$

For this difference to have the same magnitude as $\mu_{1}(h)$ would require our putting

$$
h \approx \frac{7}{27 \pi}
$$

4. The unit circle. Let $R$ be the the unit circle. Its first eigenvalue and eigenfunction are

$$
\begin{aligned}
& \lambda_{1} \approx 2.4^{2}, \\
& u_{1}=\left[\sqrt{\pi} J_{1}\left(\sqrt{\lambda_{1}}\right)\right]^{-1} J_{0}\left(r \sqrt{\lambda_{1}}\right) .
\end{aligned}
$$

Let the vector field $\left(f^{1}, f^{2}\right)$ be given by

$$
\begin{aligned}
& f^{1}=x, \\
& f^{2}=y .
\end{aligned}
$$

so that

$$
\begin{aligned}
\tau & =0, \\
\min _{\sigma} f^{i} \nu^{i} & =1, \\
\max _{R} \sqrt{f^{i} f^{i}} & =1 .
\end{aligned}
$$

Again a direct computation yields

$$
\begin{aligned}
& B_{1} \approx 38.4 \alpha^{3}+O(h), \\
& \bar{B}_{1} \approx 1.7 \alpha^{3}+O(h) .
\end{aligned}
$$

It is clear from these two examples that bounds of this paper are not sufficiently sharp to be used in the actual computation of eigenvalues. In fact, the finite difference problem posed appears far from optimal. It is hoped, however, that the techniques employed here may be useful in obtaining bounds by other finite difference approximations.

\section{BIBLIOGRAPHY}

1. N. Aronszajn, The Rayleigh-Ritz and the Weinstein methods for approximation of eigenvalues, Oklahoma A. and M. College Tech. Reports 1-4 (1949-1950).

2. - Approximation methods for eigenvalues of completely continuous symmetric operators, Proc. Symposium on Spectral Theory and Differential Problems, Oklahoma A. and M. College (1951) 179-202.

3. N. W. Bazley, Lower bounds for eigenvalues. University of Maryland dissertation.(1959). 4. - Lower bounds for eigenvalues with applications to the helium atom. Proc. Nat. Acad. Sci., 45 (1959). 
5. (1959) abstract 557-29.

6. G. Bertram, Fehlerabschätzung für das Ritz-Galerkinsche Verfahren bei Eigenwertproblemen, Z. A. M. M., 37 (1957) 191-201.

7. W. Börsch-Supan, Obere Schranken für den gröszten Eigenwert eines vollstetigen selbstadjungierten Operators, Math. Ann., 134 (1958), 453-457.

8. L. Collatz, Konvergenz des Differenzverfahrens bei Eigenwertproblemen partieller Differentialgleichungen, Deutsche Math., 3 (1938), 200-212.

9. _ Schrittweise Näherungen bei Integralgeichungen und Eigenwertschranken, Math. Zeit., 46 (1940), 692-708.

10. , Eigenwertprobleme und ihre numerische Behandlung, Chelsea, New York (1948).

11. R. Courant, Über die Eigenwerte bei den Differentialgleichungen der mathematischen Physik, Math. Zeit., 7 (1920), 1-57.

12. R. Courant and D. Hilbert, Methoden der mathematischen Physik, Berlin (1937).

13. R. Courant, Variational methods for the solutions of problems of equilibrium and vibrctions, Bull. Amer. Math. Soc., 49 (1943), 1-23.

14. J. B. Diaz, Upper and lower bounds for quadratic functionals, Proc. Symp. Spectral Theory and Diff. Probs., Oklahoma A. and M. (1950), 279-289; see also paper with same title in Collectanea Math., 4 (1951), 3-50.

15. Upper and lower bounds for eigenvalues, Proc. of the Eighth Symposium on Applied Mathematics of the A. M. S. (Calculus of Variations and its Applications) (1956), 53-78.

16. D. M. Eidus, On the solution of boundary problems by the method of finite differences, Doklady Akad. Nauk SSSR (N.S.) 83, 191-194, (1952), (Russian).

17. - On the continuous dependence of characteristic functions on the region, Doklady Akad. Nauk SSSR (N.S.) 83, 365-367, (1952), (Russian).

18. G. Faber, Beweis, dass unter allen homogenen Membranen von gleicher Fläche und gleicher Spannung die kreisförmige den tiefsten Grundton gibt, Bayrische Akad. de Wiss. Sitzungsber, (1923), 169-172.

19. G. E. Forsythe, Asymptotic lower bounds for the frequencies of certain polygonal membranes, Pacific J. Math., 4 (1954), 467-480.

20. - Asymptotic lower bounds for the fundamental frequency of convex membranes, Pacific J. Math., 5 (1955), 691-702.

21. - Difference methods on a digital computer for Laplacian boundary value and eigenvalue problems, Comm. Pure Appl. Math., 9 (1956), 425-434.

22. S. Gould, Variational methods for eigenvalue problems. An introduction to the methods of Rayleigh-Ritz, Weinstein, and Aronszajn, Math. Expositions 10, Toronto (1957).

23. A. Hammerstein, Eine Restabsclätzung für das Ritzsche Verfahren bei gewissen Variationsproblemen mit Nebenbedingungen, Sitzungsber. d. Berliner Math. Ges., 26 (1927), 171-177.

24. J. Hersch, Équations différentielles et fonctions des cellules. C. R. Acad. Sci. Paris, 240 (1955), 1602-1604

25. - Une interprétation du principe de Thomson et son analogue pour la fréquence fondamentale d'une membrane. Application. C. R. Acad. Sci. Paris, 248 (1959) 2060-2062. 26. W. Hooker and M. H. Protter, Bounds for the first eigenvalue of a rhombic membrane, Tech. Report No. 3 prepared under contract AF 49(638)-398, University of California, Berkeley (1959). To appear in J. of Math. and Phys.

27. L. Hörmander, Uniqueness theorems and estimates for normally hyperbolic partial differential equations of second order, Tolfte Skandinavska Matematikerkongressen Lund, (1953), 105-115.

28. T. Kato, On the upper and lower bounds for eigenvalues, J. Phys. Soc. Japan 4 (1949), 415-438.

29. T. Kato, H. Fujita, Y. Nakata and M. Newman, Estimation of the frequencies of thin 
elastic plates with free edges, J. of Research of the National Bureanu of Standards, $\mathbf{5 9}(1957)$, 169-186.

30. F. Koehler, Estimates for the eigenvalues of in finite matrices, Pacific, J. Math., 7 (1957), 1391-1404.

31. W. Kohn, A note on Weinstein's Variational Method, Phys. Rev., 71 (1947), 902-904. 32. E. Krahn, Über eine von Rayleigh formulierte Minimaleigenschaft des Kreises., Math. Ann., 94 (1924), 97-100.

33. E. Kreyszig, Die Einschliessung von Eigenwerten hermitescher Matrizen beim Iterationsverfahren, Z. A. M. M. 34 (1954), 459-469.

34. - Die Ausnutzung zusätzlicher Vorkenntnisse für die Einschliessung von Eigenwerten beim Iterationsverfahren, Z. A. M. M., 35 (1955), 89-95.

35. N. Krylov and N. Bogoliubov, Sur le calcul des racines de la transcendante de Fredholm les plus voisines d'une nombre donné par les méthodes des moindres carrés et de l'algorithme variationel, Isvestia Akad. Nauk SSSR, Leningrad (1929), 471-488.

36. N. Krylov, Les méthodes de solution approchée des problèmes de la physique mathématique, Mem. Sci. Math., 49 (1931).

37. C. Lanczos, Applied Analysis, Prentice Hall (1956).

38. N. J. Lehmann, Beiträge zur numerischen Lösung Linearer Eigenwertprobleme. Z. A. M. M., 29 (1949), 341-356 and 30 (1950), 1-16.

39. H. J. Maehly, Ein neues Variationsverfahren zur genäherten Berechnung der Eigenwerte hermitescher Operatoren, Helv. Phys. Acta, 25 (1952), 547-568.

40. M. Parodi, Sur quelques propriétés des valeurs charactéristiques des matricescarrés, Mem. Sci. Math., 118 (1952).

41. L. E. Payne, Inequalities for eigenvalues of membranes and plates, J. Rational Mech. and Anal., 4 (1955), 517-529.

42. L. E. Payne and H. F. Weinberger, New bounds in harmonic and biharmonic problems, J. Math. Phys., 33 (1955), 291-307.

43. - A generalized Rellich identity, Bull. Amer. Math. Soc., 63 (1957), 121.

44. - Lower bounds for vibration frequencies of elastically supported membranes and plates, J. Soc. for Ind. and Appl. Math., 5 (1957), 171-182.

45. - New bounds for solutions of second order elliptic partial differential equations, Pacific J. Math., 8 (1958), 551-573.

46. Poincaré, Sur les équations aux derivées partielles de la physique mathématique, Amer. J. of Math., 12 (1890). 211-294.

47. G. Pólya, Sur une interprétation de la méthode des differences finies qui peut fournir des bornes supérieures ou inférieures, C. R. Acad. Sci., Paris, 235 (1952), 995-997.

48. G. Pólya and M. Schiffer, Convexity of functionals by transplantation, J. d'Analyse Math., 3 (1953-54), 245-345.

49. G. Pólya, Torsional rigidity, principal frequency, electrostatic capacity, and symmetrization. Quart. of Appl. Math., 6 (1948), 217-277.

50. G. Pólya and G. Szego, Isoperimatric Inequalities in Mathematical Physics, Annals of Math. Studies, 27, Princeton University Press.

51. M. H. Protter, Lower bounds for the first eigenvalue of Elliptic Equations, Ann. of Math., 71 (1960), 423-444.

52. F. Rellich, Darstellung der Eigenwerte von $\Delta u+\lambda u$ durch ein Randintegral, Math. Z., 46 (1940), 635-636.

53. W. Ritz, Theorie der transversalschwingungen einer quadratischen Platte mit frien Rändern, Ann. d. Physik, 28 (1909), 737-786.

54. E. Saibel, Free and forced vibrations of composite systems, Proc. Symposium on Spectral Theory and Differential Problems, Oklahoma A and M College, (1951), 333-343.

55. J. L. Synge and A. Schild, Tensor Calculus, Toronto Press, 1949.

56. O. Taussky, Contributions to the solution of systems of linear equations and the determination of eigenvalues, (Nat. Standards Applied Math. Series, 39) U. S. Govt. Print. Off., 〈1954). 
57. G. Temple and W. G. Bickley, Rayleigh's principle and its applications to engineering, London 1933.

58. E. Trefftz, Über Fehlerabschätzung bei Berechnung von Eigenwerten, Math. Ann., 108 (1933), 595-604.

59. L. de Vito, Sugli autovalori e sulle autosoluzioni di una classe di trasformazioni hermitiane, Rend Sem. Mat. Padova, 25 (1956), 144-175.

60. A. G. Walker and J. D. Weston, Inclusion theorems for the eigenvalues of a normal matrix, J. London Math. Soc., 24 (1949), 28-31.

61. K. Washizu, Geometrical representations of bounds of eigenvalues, J. Japan Soc. for Appl. Mech., 5 (1952-53), 29-32.

62. , On the bounds of eigenvalues, Quart. J. of Mech. and Appl. Math., 8 (1955), 311-325.

63. H. F. Weinberger, A Rayleigh-Ritz procedure with arbitrary error estimate for differential operators with finite perturbations, (Abstract) Bull, A. M. S., 60 (1954), 348.

64. - $\dot{A}$ Rayleigh-Ritz procedure for unbounded perturbations, (Abstract) Bull. A. M.S., 60 (1954), 550.

65. - A Rayleigh-Ritz procedure giving upper and lower bounds for eigenvalues, Inst. for Fluid Dyn. and Appl. Math. U. of Maryland. Tech. Note BN-14 (1954).

66. - Upper and lower bounds for eigenvalues by finite difference methods, Comm. Pure Appl. Math., 9 (1956), 613-623. (Also, Proc. Conf. on Partial Differential Equations, Berkeley, 1955).

67. - Lower bounds for higher eigenvalues by finite difference methods. Pacific J. Math., 8 (1958) 339-368.

68. , An extension of the classical Sturm-Liouville theory, Duke Math. J., 22 (1955), 1-14.

69. , A theory of lower bounds for eigenvalues, Tech. Note BN-183, Inst. for Fluid Dyn. and Appl. Math. University of Maryland, August 1959.

70. A. Weinstein, Sur la stabilité des plaques encastrées, C. R. Acad. Sci. Paris, 200 (1935), 107-109.

71. Etude des spectres des équations aux derivées partielles de la théorie des plaques élastiques, Mem. des Sci. Math., 88, Paris 1937.

72. - Quantitative methods in Sturm-Liouville theory, Proc. Symposium on Spectral Theory and Differential Problems. Okla. A and M College, (1951), 345-352.

73. - Variational methods for the approximation and exact computation of eigenvalues, Nat. Breau of standards Appl. Math. Ser., 29 (1953), 83-89.

74. D. H. Weinstein, Modified Ritz method, Proc. Nat. Acad. Sci. U. S. A. 20 (1934), 529-532.

75. H. Wielandt, Inclusion theorems for eigenvalues, Nat. Bureau of Standards Appl. Math. Ser., 29 (1953), 75-78.

76. - Einschliessung von Eigenwerten Hermitescher Matrizen nach dem Abschnittsverfahren, Arc. d. Mathematik, 5 (1954), 108-114.

U. S. Naval ORdnance Laboratory White OAK, Silver Spring, Maryland 


\title{
PACIFIC JOURNAL OF MATHEMATICS
}

\author{
EDITORS
}

\author{
Ralph S. Phillips \\ Stanford University \\ Stanford, California \\ F. H. Brownell \\ University of Washington \\ Seattle 5, Washington
}

\author{
A. L. Whiteman \\ University of Southern California \\ Los Angeles 7, California \\ L. J. PAige \\ University of California \\ Los Angeles 24, California
}

ASSOCIATE EDITORS
E. F. BECKENBACH
D. DERRY
H. L. ROYDEN
E. G. STRAUS
T. M. CHERRY
M. OHTSUKA
E. SPANIER
F. WOLF

\section{SUPPORTING INSTITUTIONS}

\author{
UNIVERSITY OF BRITISH COLUMBIA \\ CALIFORNIA INSTITUTE OF TECHNOLOGY \\ UNIVERSITY OF CALIFORNIA \\ MONTANA STATE UNIVERSITY \\ UNIVERSITY OF NEVADA \\ NEW MEXICO STATE UNIVERSITY \\ OREGON STATE COLLEGE \\ UNIVERSITY OF OREGON \\ OSAKA UNIVERSITY \\ UNIVERSITY OF SOUTHERN CALIFORNIA
}

\author{
STANFORD UNIVERSITY \\ UNIVERSITY OF TOKYO \\ UNIVERSITY OF UTAH \\ WASHINGTON STATE COLLEGE \\ UNIVERSITY OF WASHINGTON \\ * * * * \\ AMERICAN MATHEMATICAL SOCIETY \\ CALIFORNIA RESEARCH CORPORATION \\ HUGHES AIRCRAFT COMPANY \\ SPACE TECHNOLOGY LABORATORIES \\ NAVAL ORDNANCE TEST STATION
}

Mathematical papers intended for publication in the Pacific Journal of Mathematics should be typewritten (double spaced), and the author should keep a complete copy. Manuscripts may be sent to any one of the four editors. All other communications to the editors should be addressed to the managing editor, L. J. Paige at the University of California, Los Angeles 24, California.

50 reprints per author of each article are furnished free of charge; additional copies may be obtained at cost in multiples of 50 .

The Pacific Journal of Mathematics is published quarterly, in March, June, September, and December. The price per volume (4 numbers) is $\$ 12.00$; single issues, $\$ 3.50$. Back numbers are available. Special price to individual faculty members of supporting institutions and to individual members of the American Mathematical Society: $\$ 4.00$ per volume; single issues, $\$ 1.25$.

Subscriptions, orders for back numbers, and changes of address should be sent to Pacific Journal of Mathematics, 103 Highland Boulevard, Berkeley 8, California.

Printed at Kokusai Bunken Insatsusha (International Academic Printing Co., Ltd.), No. 6, 2-chome, Fujimi-cho, Chiyoda-ku, Tokyo, Japan.

\section{PUBLISHED BY PACIFIC JOURNAL OF MATHEMATICS, A NON-PROFIT CORPORATION}

The Supporting Institutions listed above contribute to the cost of publication of this Journal, but they are not owners or publishers and have no responsibility for its content or policies. 


\section{Pacific Journal of Mathematics}

\section{Vol. 11, No. 2 December, 1961}

Tsuyoshi Andô, Convergent sequences of finitely additive measures........

Richard Arens, The analytic-functional calculus in commutative topological algebras..........................................

Michel L. Balinski, On the graph structure of convex polyhedra in

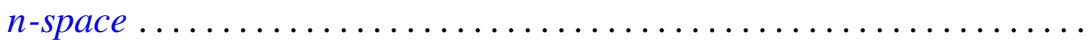

R. H. Bing, Tame Cantor sets in $E^{3}$...

Cecil Edmund Burgess, Collections and sequences of continua in the plane.

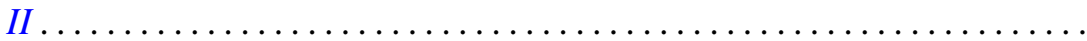

J. H. Case, Another 1-dimensional homogeneous continuum which contains an $\operatorname{arc}$

Lester Eli Dubins, On plane curves with curvature ................. 471

A. M. Duguid, Feasible flows and possible connections .............. 483

Lincoln Kearney Durst, Exceptional real Lucas sequences ................ 489

Gertrude I. Heller, On certain non-linear opeartors and partial differential equations........................................

Calvin Virgil Holmes, Automorphisms of monomial groups

Wu-Chung Hsiang and Wu-Yi Hsiang, Those abelian groups characterized by their completely decomposable subgroups of finite rank ..........

Bert Hubbard, Bounds for eigenvalues of the free and fixed membrane by finite difference methods .........................

D. H. Hyers, Transformations with bounded mth differences. .

Richard Eugene Isaac, Some generalizations of Doeblin's decomposition

John Rolfe Isbell, Uniform neighborhood retracts ..........

Jack Carl Kiefer, On large deviations of the empiric D. F. of vector chance variables and a law of the iterated logarithm...

Marvin Isadore Knopp, Construction of a class of modular functions and forms. II. . .

Gunter Lumer and R. S. Phillips, Dissipative operators in a Banach space....

Nathaniel F. G. Martin, Lebesgue density as a set function ...

Shu-Teh Chen Moy, Generalizations of Shannon-McMillan theorem ...

Lucien W. Neustadt, The moment problem and weak convergence in $L^{2}$

Kenneth Allen Ross, The structure of certain measure algebras...

James F. Smith and P. P. Saworotnow, On some classes of scalar-product algebras.

Dale E. Varberg, On equivalence of Gaussian measures. . 